\title{
Performance improvement of an extraction Lambertson septum magnet in the Spallation Neutron Source accumulator ring
}

\author{
J. G. Wang* \\ Oak Ridge National Laboratory, Oak Ridge, Tennessee 37831, USA
}

(Received 3 December 2008; published 29 April 2009)

\begin{abstract}
The Spallation Neutron Source ring extraction Lambertson septum magnet contains a strong skew quadrupole term, which has been identified as the source of causing a beam profile distortion on the target. We have performed 3D computer simulations to study the magnetic field quality in the magnet. The skew quad term is computed with different methods in simulations and is compared to measurement data. The origin of the large skew quad term is thoroughly investigated. The remedy for minimizing the skew quad term by modifying the magnet is proposed. Particle tracking is performed to verify the beam profile evolution through the existing and modified septum. The magnetic interference to the septum performance from an adjacent quadrupole is also assessed.
\end{abstract}

DOI: 10.1103/PhysRevSTAB.12.042402

PACS numbers: 03.50.-z, 29.27.Ac, 41.85.Ew, 85.70.-w

\section{INTRODUCTION}

The Spallation Neutron Source (SNS) accumulator ring extraction system is built in one of its four straight sections. Figure 1 shows a part of the extraction straight section in top view. It contains an extraction Lambertson septum (ELS) magnet followed by a quadrupole doublet assembly. During the ring accumulation, a circulating proton beam passes through a well-shielded aperture within the upper yoke of the ELS. Its effect on the beam should be negligible. The ring extraction takes place in a single turn and two steps after the beam is fully accumulated. Four pulsed kickers upstream (not shown in figure) are first fired. This pushes the beam down to the dipole entrance of the ELS, which in turn bends the beam horizontally out of the ring.

The ELS was designed and developed at Brookhaven National Lab (BNL) [1-3]. Its structure is quite complex and its field qualities are very critical to the extracted beam as well as to the circulating beam. Though the ELS extraction line was measured with a flip coil on grids before delivery, there were no official test data of harmonic contents provided.

Since the SNS ring commissioning, we have found that the extracted beam profile on the target is slightly tilted, as shown in Fig. 2 [4]. This tilted beam profile does not match the target geometry and has been an issue of concern for high power operation. A considerable amount of effort has been devoted to finding the source of the beam profile tilt. Finally, 3D simulation studies of the ELS field qualities show a strong skew quadrupole term in the ELS extraction line, and this has been identified to be responsible for the beam profile tilt on the target. Since the magnet is already installed for operation, there is no way to conduct further measurements on the magnet. This has motivated us to

\footnotetext{
*jgwang@ornl.gov
}

carefully model the magnet in 3D computer simulations in order to accurately calculate the skew quad term and to improve its field quality by minimizing the skew quad term.

In this paper we first introduce our simulation model of the ELS in Sec. II. Its general performance characteristics for the circulating beam and extracted beam are reported in Sec. III. The computation of the skew quad term with three different methods and a comparison with the BNL measurement data are detailed in Sec. IV. In Sec. V we investigate the origin of the large skew quad component. This is caused by the lack of midplane symmetry of the magnet. The contributions to the skew quad term from the ELS central region and the two ends are analyzed. In Sec. VI the remedies for minimizing the skew quad term are proposed. Section VII provides a beam tracking study, which shows clearly a beam profile tilt in the existing ELS and its elimination in the modified one. The magnetic interference to the septum performance from an adjacent quadrupole is assessed in Sec. VIII. A short summary can be found in Sec. IX.

\section{3D SIMULATION MODEL}

The simulation environment in this work is OPERA-3D/ TOSCA v. 12 [5]. The version 12 can operate in computers with 64 bits and allows the users to employ unlimited

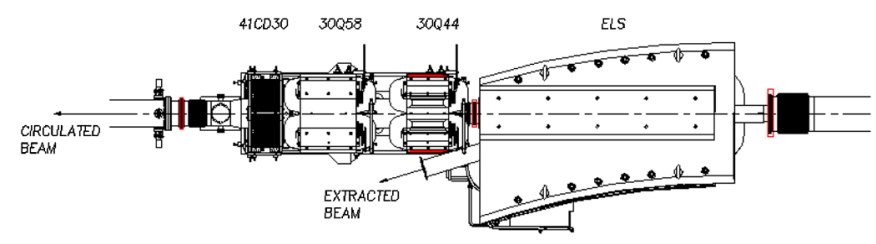

FIG. 1. (Color) ELS and quad assembly (courtesy of BNL design drawing and specifications). 


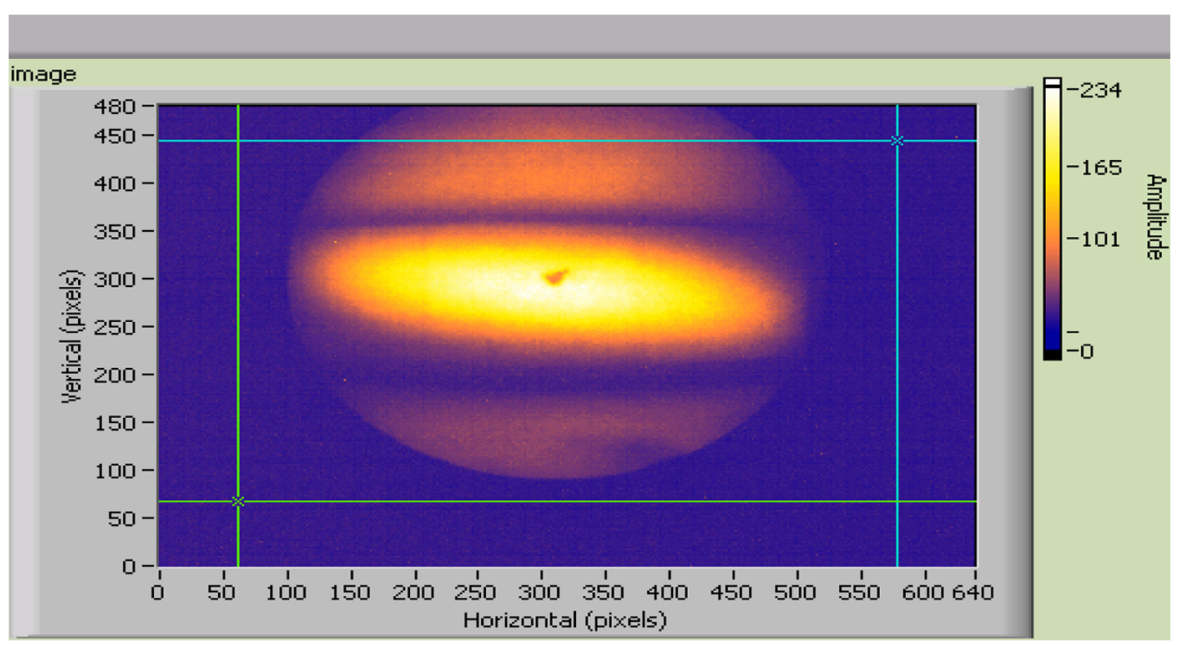

FIG. 2. (Color) Beam image from the target view screen [4], where the beam size is about $20 \mathrm{~cm}$ by $7 \mathrm{~cm}$.
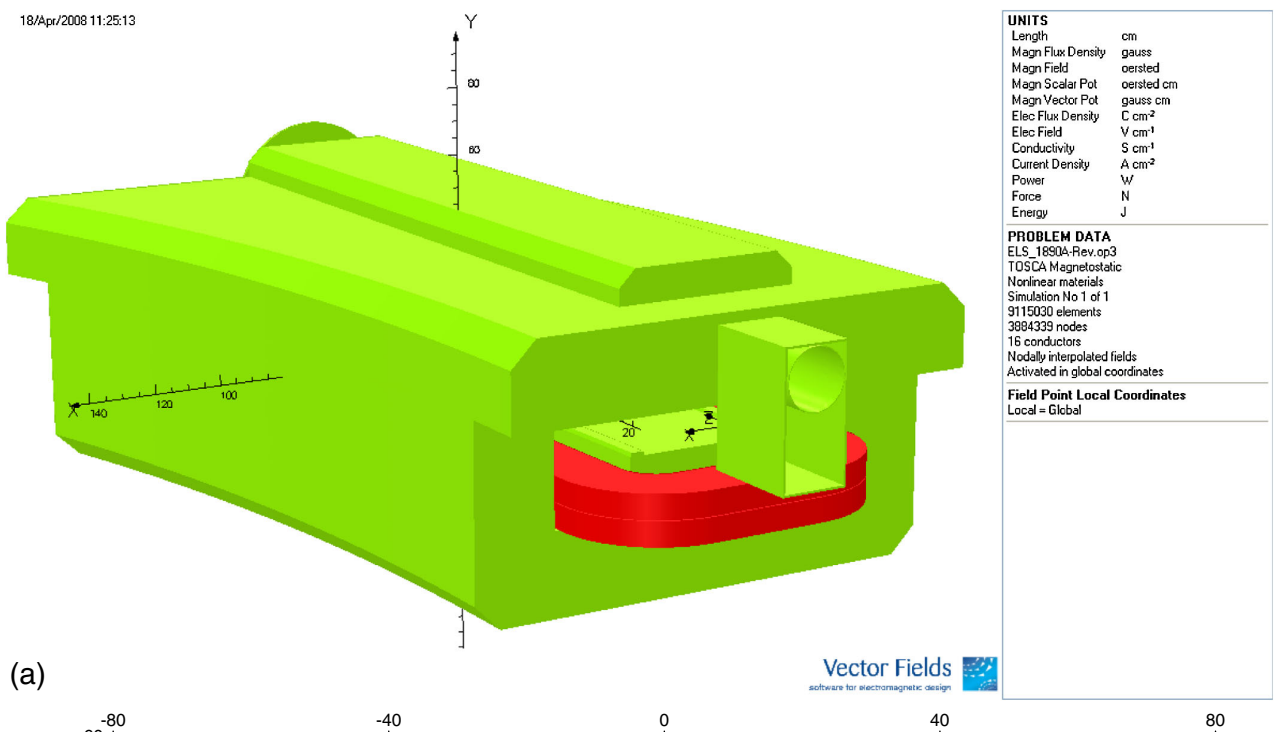

(a)

Vector Fields 䜾

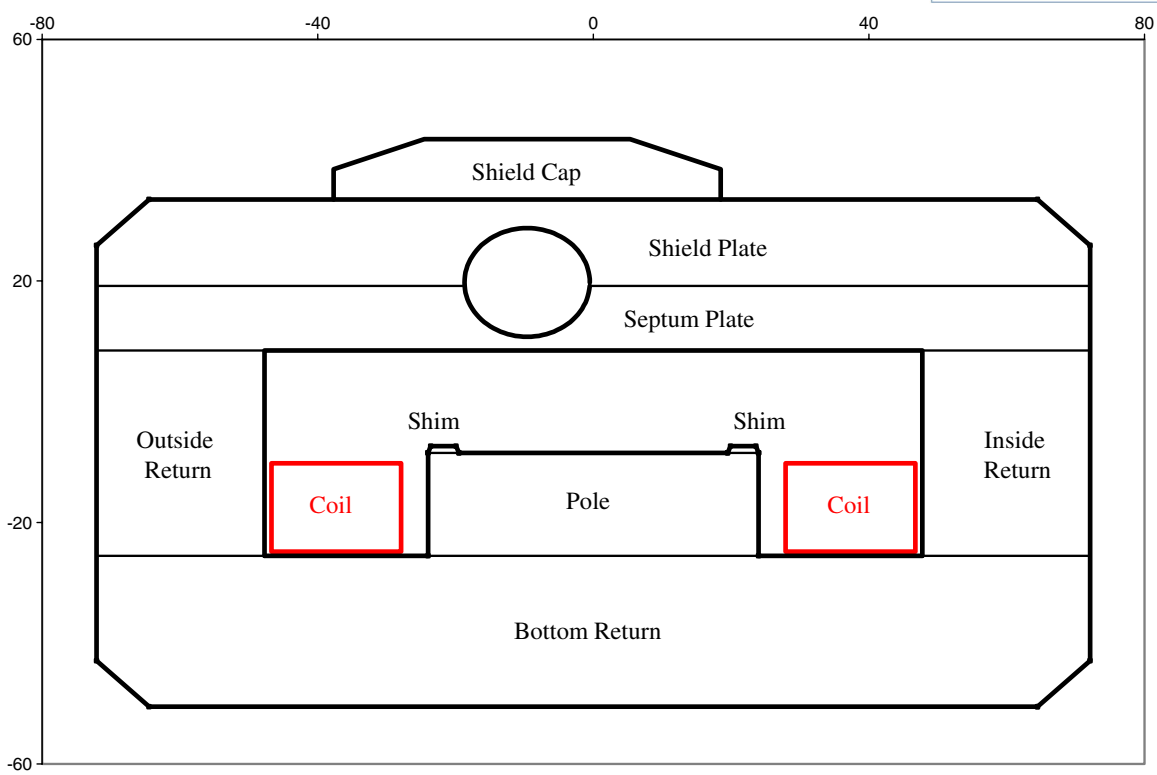

FIG. 3. (Color) (a) Entrance view of ELS simulation model. (b) Upstream view of a cross section through the center of ELS model. 
TABLE I. ELS main design parameters [1-3].

\begin{tabular}{lc}
\hline \hline Pole length (m) & 2.23063 \\
Pole width (m) & 0.48006 \\
Septum plate length (m) & 2.63042 \\
Septum plate width (m) & 1.44196 \\
Dipole gap for extracted beam (m) & 0.16988 \\
Effective (hard edge) length (m) & 2.44 \\
Bending angle (degree) & 16.8 \\
Bending field @ 1 GeV (T) & 0.68 \\
Aperture for circulating beam (m) & 0.175 \\
\hline \hline
\end{tabular}

elements in models. The ELS model is shown in Fig. 3(a). A cross section at the magnet center and in perpendicular to the extracted beam axis is shown in Fig. 3(b). The model is built with the OPERA package "Modeller" instead of the "Pre-processor". This makes it easier to study the interference between two magnets. In the model, mechanical dimensions follow the BNL design drawings and specifications, as listed in Table I. The circulating beam passes through the upper, round, straight aperture, which is very well shielded by the septum plate and the end structures. The extracted beam goes through the lower, curved gap between the ELS pole tips. On the pole tip two longitudinal shims are added to improve the bending field uniformity. The ELS is installed with a roll angle of $2.55^{\circ}$ and a pitch angle of $0.53^{\circ}$ in order to accommodate the vertical velocity of the extracted beam at the magnet entrance. These features are neglected in the simulation, but this should not affect the field quality study.

In the model, the coordinate system origin is that $z=0$ is at $12.116 \mathrm{~cm}$ away from the pole-tip end on the magnet entrance side, $y=0$ is at the midplane of the bending dipole, and $x=0$ is at the circulating beam axis. The magnet is energized at $1890 \mathrm{~A}$ in the model, which is a nominal design value for a $1 \mathrm{GeV}$ proton beam. The model consists of fine meshes for accurate results. It contains approximately ten million elements and its postprocessor file occupies more than 2 GB memory. The B-H curve used in the simulations is a default one in OPERA-3D code. The septum is constructed with low carbon steel AISI 1006, but its exact B-H curve is not available. We believe that the error thus introduced for the main field should be less than a quarter percent according to our previous experience with other SNS magnet simulations and measurements.

\section{GENERAL PERFORMANCE OF ELS}

The SNS ring extraction septum combines two general functions in one magnet. First, it provides a straight path inside the upper yoke for the circulating beam. The main criterion for this function is to make the magnetic field along the path a minimum. The beam aperture is $17.5 \mathrm{~cm}$ and the septum thickness is $1 \mathrm{~cm}$. The circulating beam pipe is made of 1010 steel, and about $1 \mathrm{~mm}$ copper shielding is placed between the pipe and the magnet iron. In order to reduce the effect of the dipole fringe field below, the septum plate is made approximately $20 \mathrm{~cm}$ longer than the pole tip at each end. In addition, a shielding box is attached to the septum entrance face, and a round cap of iron is attached to the septum exit face. Second, the magnet bends the beam horizontally out of the ring during the extraction process. The main bending parameters, such as the bending angle, the nominal bending field, and the effective (hard edge) length, are listed in Table I. Good field quality is essential for the performance of the extracted beam. Especially, the skew quad term should be small enough in order not to distort the extracted beam profile.

Figure 4 shows a zone map of the total magnetic flux density $B_{\text {mod }}$ across the circulating beam aperture at the magnet longitudinal center $(z=123.428 \mathrm{~cm})$. The field amplitude at different locations is indicated by the color index bar on the left side. Within the beam aperture, the magnetic field is at the $0.5 \mathrm{G}$ level. The field amplitude in the surrounding yoke is as high as more than 1.2 T. This demonstrates the very effective shield of the magnetic beam pipe with a surrounding gap of $1.181 \mathrm{~mm}$, in addition to the reduction of residual field in the circulating beam aperture of a Lambertson septum structure.

The magnetic field distribution along the circulating beam axis in the ELS is shown in Fig. 5. Although a shielding box at the septum entrance and a round cap of iron at its exit are applied, the fringe field on the circulating beam axis in these regions still does not vanish. The integrated $B_{y}$ component is about $-0.0027 \mathrm{~T}-\mathrm{m}$, while the integrated $B_{x}$ is $0.0012 \mathrm{~T}-\mathrm{m}$. This results in a total dipole field of $0.0029 \mathrm{~T}-\mathrm{m}$, which can be compensated by dipole correctors downstream.

By employing a rotation patch, which has the dimensions of $r=-4.191$ to 8.262 and $z=-200$ to $500 \mathrm{~cm}$ as explained later in Sec. IVA, we can evaluate the field within the circulating beam aperture. The method yields an integrated dipole field of $0.0029 \mathrm{~T}-\mathrm{m}$ with a phase angle of $-157^{\circ}$. These are in fact the same values as those obtained on the circulating beam axis, as shown in Fig. 5. The integrated quad term is $0.006 \mathrm{~T}$ with a phase angle of $65.9^{\circ}$. The magnetic field parameters of the ELS along the circulating beam line were also measured by BNL with a rotating coil. Their data show that the integrated dipole component at $1890 \mathrm{~A}$ is $0.0032 \mathrm{~T}-\mathrm{m}$, which is slightly larger than our simulated value.

For the extracted beam, the magnetic field quality is also studied in the postprocessor file. Figure 6 shows the magnetic field across the ELS gap at its longitudinal center, where the local coordinate origin is $x=9.064, y=0, z=$ $123.428 \mathrm{~cm}$, and the three Euler's angles are $0,8.4^{\circ}$, and 0 , respectively. The dipole field $B_{y}$ is very uniform within the beam aperture region with a magnitude of $6687.9 \mathrm{G}$ at its 

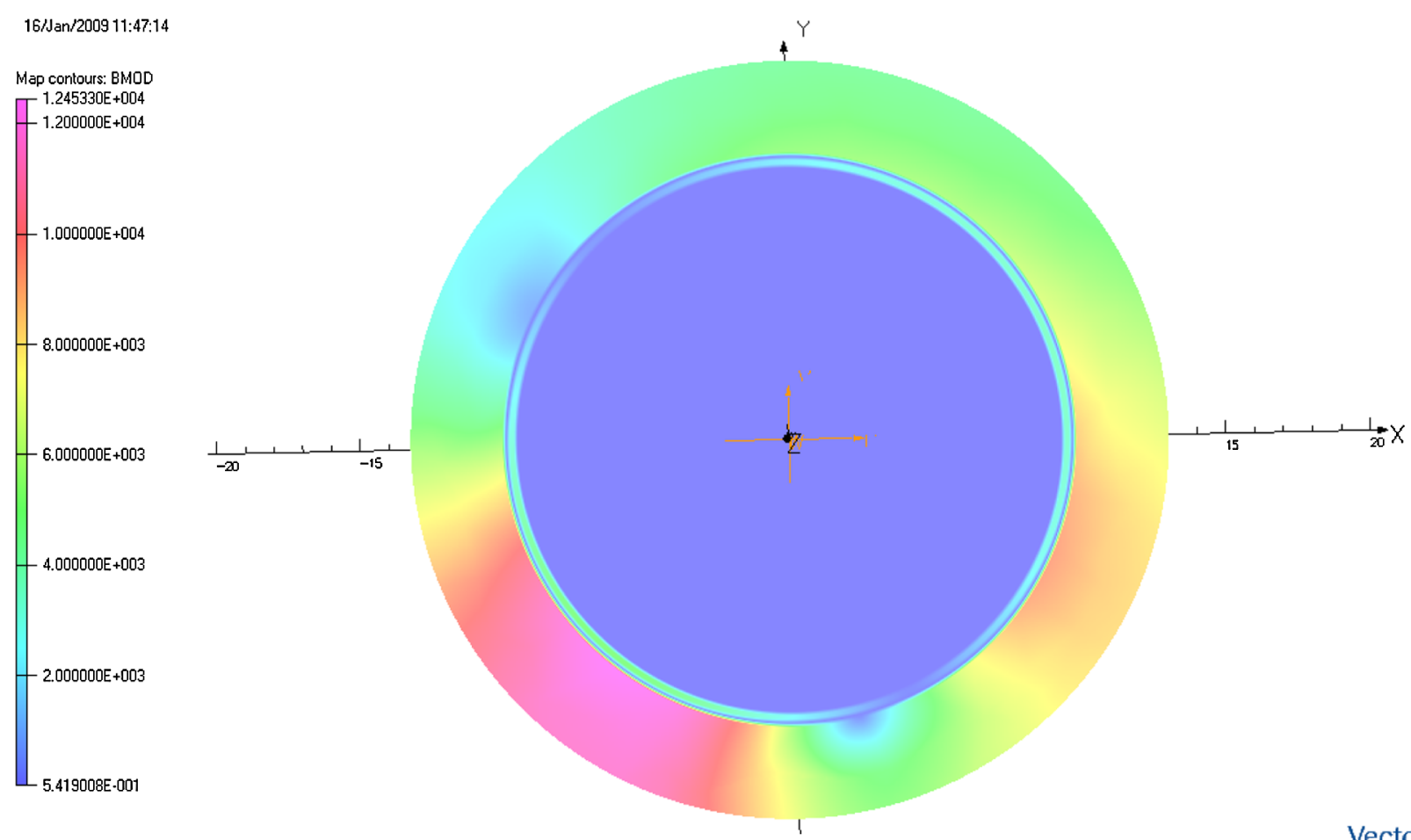

Vector Fields

FIG. 4. (Color) Magnetic field across circulating beam aperture at magnet center.

center. Figure 7 shows the magnetic bending field along a reference trajectory for the extracted beam. Inside the hard edge, the reference track follows the designed, curved ELS axis, while it is straight outside the hard edge. The total integrated dipole field along the reference trajectory is $1.692 \mathrm{~T}-\mathrm{m}$. This results in a hard edge length of $2.53 \mathrm{~m}$ and a bending angle of $17.1^{\circ}$ for $1 \mathrm{GeV}$ proton beams, which is slightly larger than the nominal value of $16.8^{\circ}$.

15/Jan/2009 15:11:59

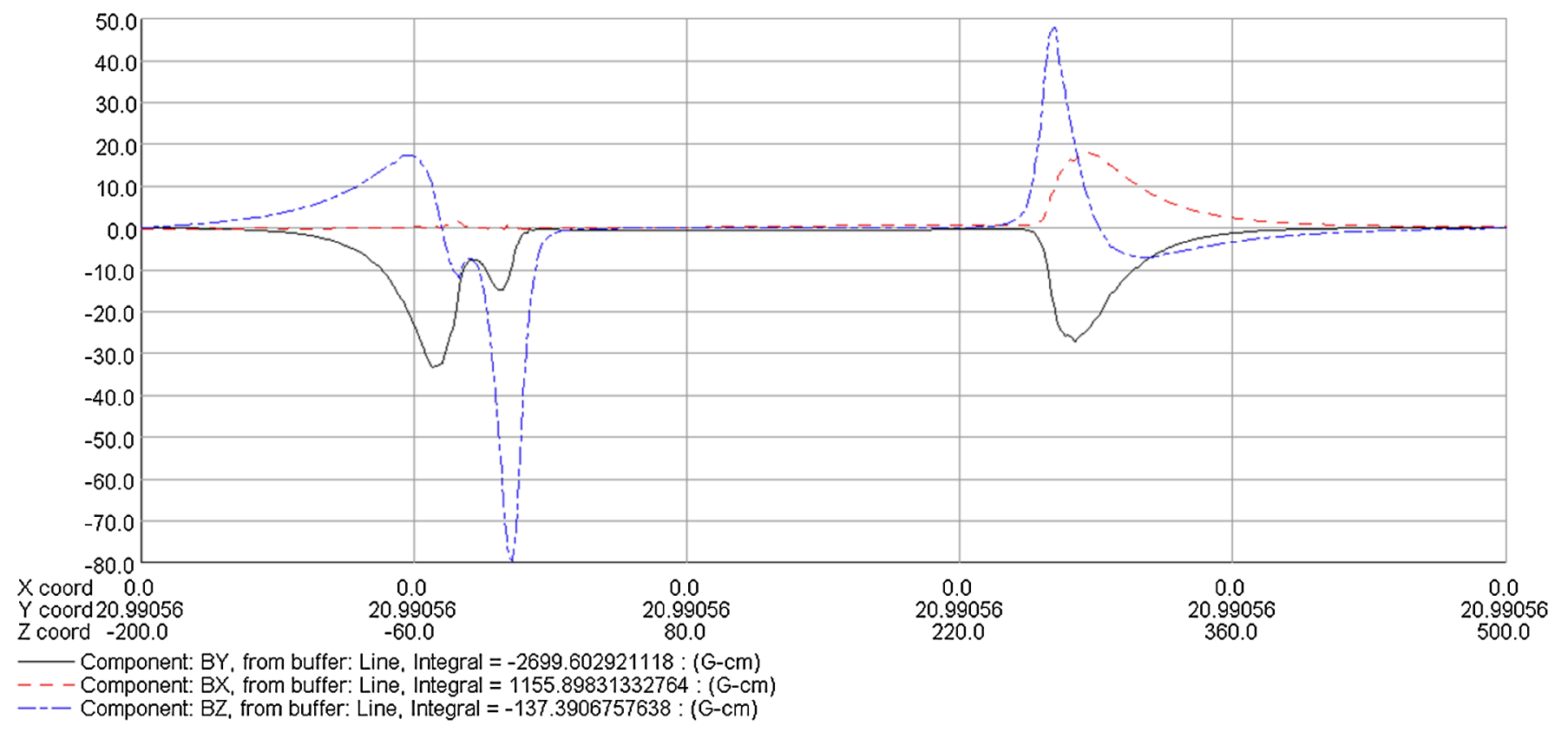

Vector Fields

FIG. 5. (Color) Magnetic field distribution on circulating beam axis. 


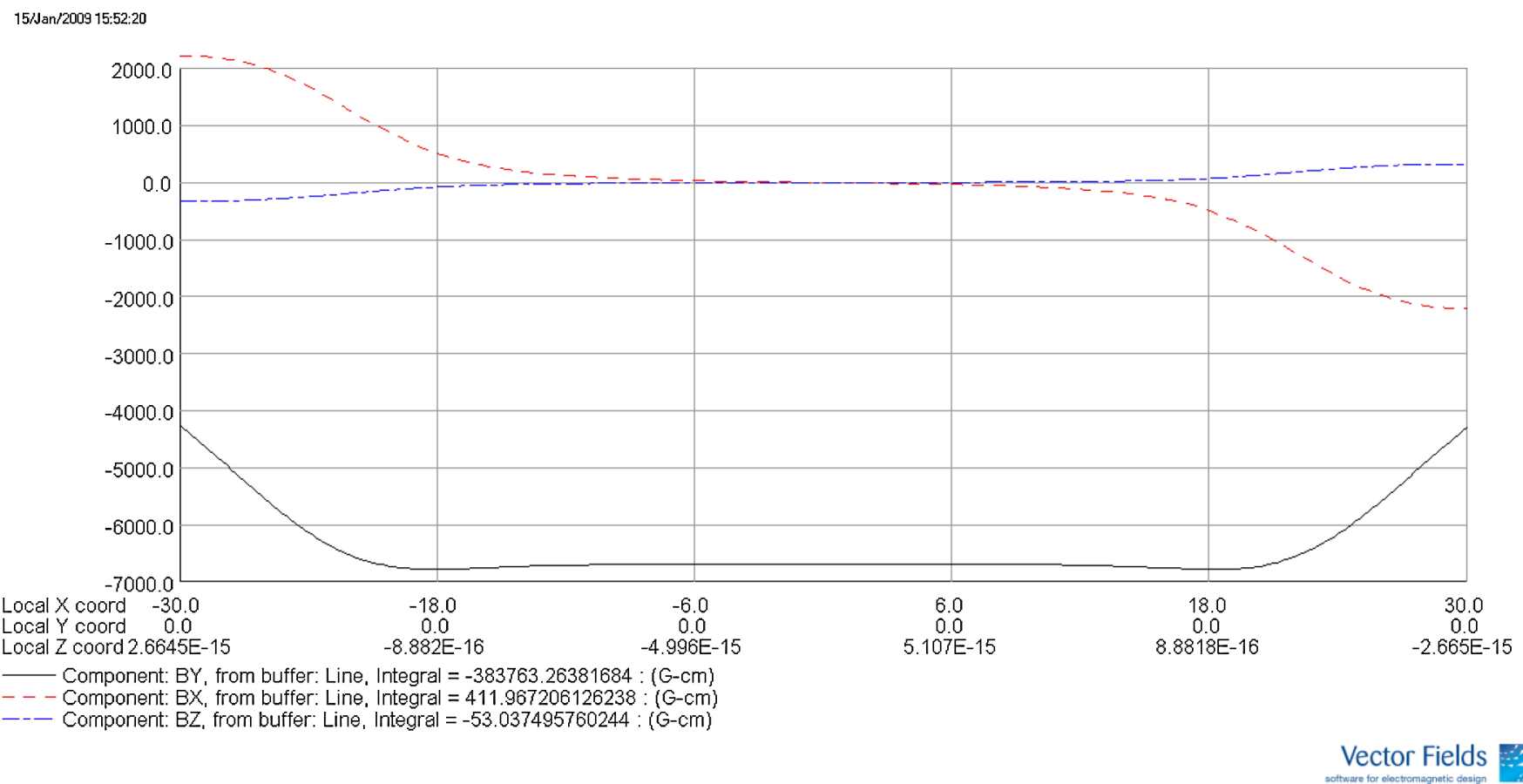

FIG. 6. (Color) Magnetic field across ELS gap at its center.

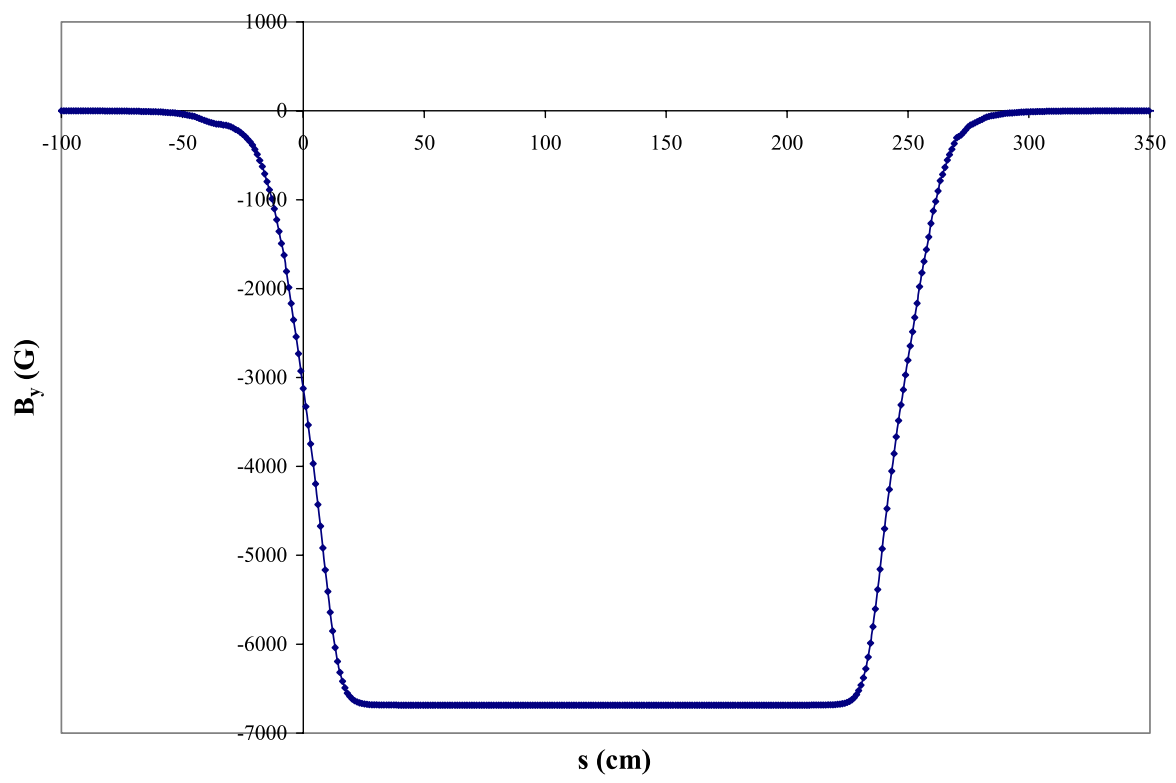

FIG. 7. (Color) Magnetic dipole field on reference trajectory for extracted beam.

\section{COMPUTATION OF SKEW QUAD TERM}

The skew quad term in the ELS extraction channel is computed with three different methods: the patch rotation, the surface field, and the dipole field on grids. Different methods are employed in computation of the same parameter because of cross-checking purposes for gaining confidence in the modeling. In addition, the simulation results can be compared directly with the BNL measurement data.

\section{A. Patch rotation method}

The patch rotation method is used to calculate the magnetic flux through a rotating Cartesian patch in a magnet. The rectangular patch is similar to an unbucked winding in a Halbach search coil in accelerator magnet measurements [6]. The patch has a radial dimension from $r_{1}$ to $r_{2}$ and a length long enough to cover all the fringe regions. It rotates about the magnet axis, so the magnetic flux is an integration of the azimuthal field component over the patch area at 
a given rotation angle. In cylindrical coordinates, the components of a two-dimensional magnetic field can be expressed by [7]

$$
\begin{aligned}
& B_{r}(r, \theta)=\sum_{m=1}^{\infty} C_{m}\left(\frac{r}{R}\right)^{m-1} \sin \left[m\left(\theta-\alpha_{m}\right)\right] \\
& B_{\theta}(r, \theta)=\sum_{m=1}^{\infty} C_{m}\left(\frac{r}{R}\right)^{m-1} \cos \left[m\left(\theta-\alpha_{m}\right)\right],
\end{aligned}
$$

where $C_{m}$ and $\alpha_{m}$ are the field amplitude and phase angle of the $2 m$-pole term of the total field, and $R$ is a reference radius within the magnet aperture. The magnetic flux through the patch can be computed as

$$
\begin{aligned}
\Phi(\theta) & =\int_{-\infty}^{+\infty} d z \int_{r_{1}}^{r_{2}} d r B_{\theta}(r, \theta) \\
& =\sum_{m=1}^{\infty}\left(C_{m} * L\right) \cos \left[m\left(\theta-\alpha_{m}\right)\right] \frac{1}{m R^{m-1}}\left(r_{2}^{m}-r_{1}^{m}\right),
\end{aligned}
$$

where $\left(C_{m} * L\right)$ represents the integrated field amplitude. By performing Fourier analysis of $\Phi(\theta)$,

$$
\mathcal{F}[\Phi(\theta)]=\sum_{k=1}^{\infty} X_{k} \angle \theta_{k},
$$

we find the integrated field amplitude and phase angle for each harmonic number $m=k$ as

$$
\begin{aligned}
C_{m} * L & =X_{k}\left(m R^{m-1}\right) /\left(r_{2}^{m}-r_{1}^{m}\right), \\
\alpha_{m} & =\theta_{k} / m .
\end{aligned}
$$

According to the "European Convention," the integrated $2 m$-pole normal and skew terms are

$$
\begin{aligned}
& B_{m} * L=\left(C_{m} * L\right) \cos \left(m \alpha_{m}\right), \\
& A_{m} * L=-\left(C_{m} * L\right) \sin \left(m \alpha_{m}\right) .
\end{aligned}
$$

Thus, the corresponding equations for the radial and the azimuthal components of the field are

$$
\begin{aligned}
& B_{r}(r, \theta)=\sum_{m=1}^{\infty}\left(\frac{r}{R}\right)^{m-1}\left[B_{m} \sin (m \theta)+A_{m} \cos (m \theta)\right] \\
& B_{\theta}(r, \theta)=\sum_{m=1}^{\infty}\left(\frac{r}{R}\right)^{m-1}\left[B_{m} \cos (m \theta)-A_{m} \sin (m \theta)\right] .
\end{aligned}
$$

For magnetic dipoles, the integrated bending field $(B *$ $\left.L=C_{1} * L\right)$ and integrated harmonics $\left(C_{m} * L, m>1\right)$ are calculated. It is common practice to express the normalized coefficients $N C_{m}$ of the integrated harmonic contents as

$$
N C_{m}=\frac{\left(C_{m} * L\right)}{\left(C_{1} * L\right)} \times 10^{4}=\frac{\left(C_{m} * L\right)}{(B * L)} \times 10^{4} \text { (units). }
$$

The rectangular patch in this calculation has a transverse dimension of $r_{1}=-4.191$ to $r_{2}=8.362 \mathrm{~cm}$, which follows the Halbach coil design criteria [6]. Since the reference trajectory inside the hard edge magnet is curved, we have to use piecewise straight patches. There are a total of six patches employed. The first patch is for the upstream region with a length of $z=-150$ to $0 \mathrm{~cm}$ and its axis coincides with the $z$ axis. The sixth one is for the downstream region with a length of $200 \mathrm{~cm}$ and starts at $x=$ $36.062, y=0$, and $z=244.208 \mathrm{~cm}$. Its axis forms an angle of $16.8^{\circ}$ with respect to the $z$ axis. Inside the hard edge magnet, the four patches uniformly divide the whole arc length. Each of the four has a length of $61.922 \mathrm{~cm}$ and their Euler's angles are phi $=p s i=0$, and theta $=2.1$, $6.3,10.5$, and 14.7 degrees, respectively. In this way, we

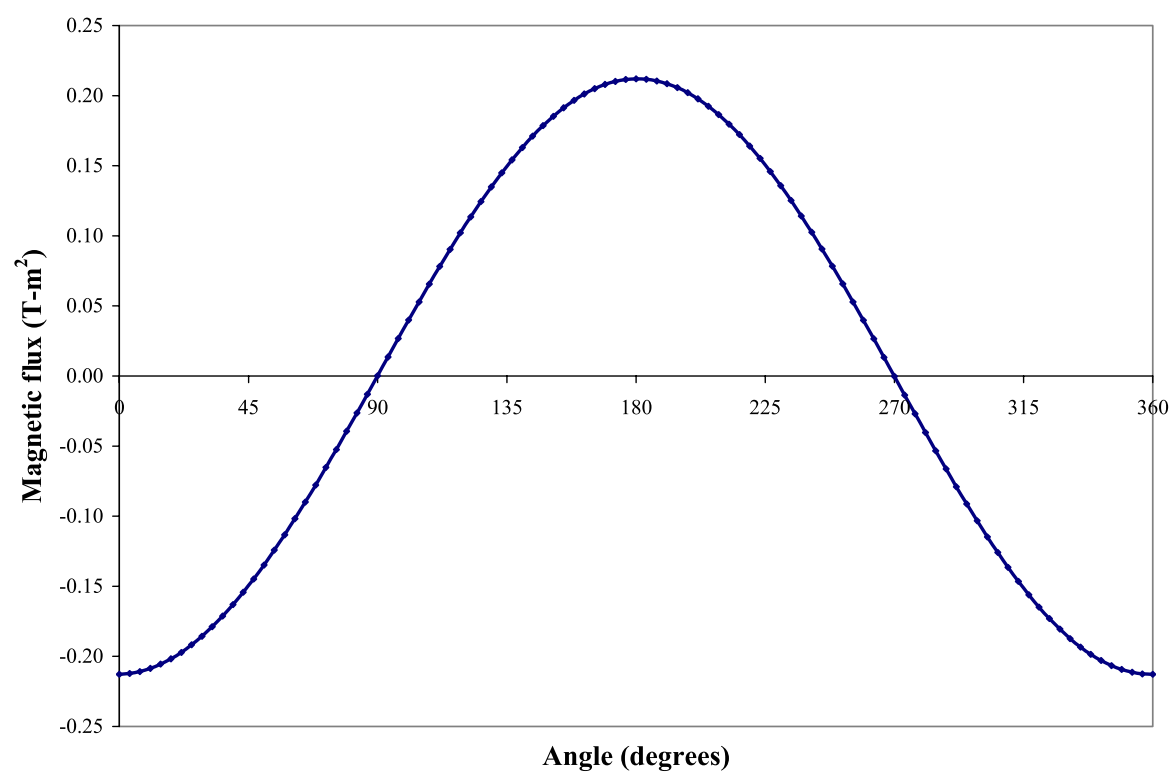

FIG. 8. (Color) Total magnetic flux through the patches vs rotation angle. 
TABLE II. Integrated ELS harmonics in T-m from various methods.

\begin{tabular}{rrrrrrrrr}
\hline \hline & \multicolumn{2}{c}{ Patch rotation } & \multicolumn{2}{c}{ Surface field } & \multicolumn{2}{c}{ Dipole field on grid } & \multicolumn{2}{c}{ BNL test } \\
$m$ & \multicolumn{1}{c}{$B_{m} * L$} & $A_{m} * L$ & \multicolumn{1}{c}{$B_{m} * L$} & $A_{m} * L$ & \multicolumn{1}{c}{$B_{m} * L$} & $A_{m} * L$ & \multicolumn{1}{c}{$B_{m} * L$} & $A_{m} * L$ \\
\hline 1 & -1.6925 & -0.0022 & -1.6926 & -0.0017 & -1.6916 & & -1.7055 & -0.0166 \\
2 & -0.0059 & -0.0214 & -0.0066 & -0.0215 & 0.0008 & -0.0221 & -0.0005 & -0.0011 \\
3 & 0.0027 & -0.0007 & 0.0027 & -0.0001 & 0.0023 & 0.0001 & 0.0077 & -0.0022 \\
4 & 0.0001 & -0.0050 & 0.0002 & -0.0050 & 0.0002 & -0.0051 & -0.0012 & -0.0001 \\
5 & 0.0001 & -0.0001 & 0.0001 & -0.0001 & 0.0002 & 0.0001 & -0.0025 & -0.0025 \\
\hline \hline
\end{tabular}

approximate each $4.2^{\circ}$ arc inside the magnet by a straight segment. We have tried more than four patches inside the hard edge magnet, but found no significant improvement of the result.

The total magnetic flux through the six patches as a function of the rotation angle is shown in Fig. 8. It looks roughly like a sinusoidal signal for a dipole. The peak flux amplitude, which happens at $\theta=0$ and $180^{\circ}$, is about $0.21 \mathrm{~T}-\mathrm{m}^{2}$. A Fourier decomposition of the signal yields all the harmonics, where a reference radius $R=8 \mathrm{~cm}$ is used. Table II shows the first five of them. The table also includes the data from the other methods described in more detail later. The integrated normal dipole term is $B_{1} * L=$ $-1.6925 \mathrm{~T}-\mathrm{m}$, which is in the minus $y$ direction and bends $1 \mathrm{GeV}$ protons by $17.14^{\circ}$, slightly higher than the designed value of $16.8^{\circ}$. These numbers are very close to those derived from Fig. 7. The integrated total quadrupole term accounts for 131 units. For a transport line magnet, the conventionally required field quality is that the higher harmonics should be less than 10 units. Thus, the total quadrupole term in the septum is more than 1 order of magnitude higher than the conventional requirement. The integrated skew quad term is $A_{2} * L=-0.0214 \mathrm{~T}$-m. Dividing the value by the radius of $R=8 \mathrm{~cm}$ yields an integrated gradient of $0.267 \mathrm{~T}$.

\section{B. Surface field analysis}

This method is employed to calculate the magnetic field component $B_{r}$ or $B_{\theta}$ on a cylindrical surface in order to obtain the $z$-dependent harmonic distribution of magnetic field along a reference trajectory. In cylindrical coordinates, the components of a three-dimensional magnetic field can be written in the form $[8,9]$

$$
\begin{aligned}
B_{r}= & \sum_{m=0} \sum_{\ell=0}^{\infty}(-1)^{\ell} \frac{m !(2 \ell+m)}{2^{2 \ell} \ell !(\ell+m) !} r^{2 \ell+m-1} \\
& \times\left[C_{m, s}^{[2 \ell]}(z) \sin (m \theta)+C_{m, c}^{[2 \ell]}(z) \cos (m \theta)\right] \\
B_{\theta}= & \sum_{m=0} \sum_{\ell=0}^{\infty}(-1)^{\ell} \frac{m ! m}{2^{2 \ell} \ell !(\ell+m) !} r^{2 \ell+m-1} \\
& \times\left[C_{m, s}^{[2 \ell]}(z) \cos (m \theta)-C_{m, c}^{[2 \ell]}(z) \sin (m \theta)\right] .
\end{aligned}
$$

Here $C_{m, s}^{[2 \ell]}(z)$ and $C_{m, c}^{[2 \ell]}(z)$ are the $2 \ell$ th derivatives of the generalized gradients $C_{m, s}(z) \equiv C_{m, s}^{[0]}(z)$ and $C_{m, c}(z) \equiv$
$C_{m, c}^{[0]}(z)$, which represent the normal and skew terms, respectively. The generalized gradients $C_{m, s}(z)$ and $C_{m, c}(z)$ for any practical magnet are a good function of $z$, and they should vanish when $z$ goes to plus or minus infinity. Thus, the integrated magnetic field at a radius $r=R$ can be expressed as

$$
\begin{aligned}
B_{r}(R) * L= & \sum_{m=1} m R^{m-1}\left[\left(C_{m, s} * L\right) \sin (m \theta)\right. \\
& \left.+\left(C_{m, c} * L\right) \cos (m \theta)\right] \\
B_{\theta}(R) * L= & \sum_{m=1} m R^{m-1}\left[\left(C_{m, s} * L\right) \cos (m \theta)\right. \\
& \left.-\left(C_{m, c} * L\right) \sin (m \theta)\right] .
\end{aligned}
$$

The integrated normal and skew terms of the integrated field are related to the generalized gradient $C_{m, s}$ and $C_{m, c}$ in a 3D multipole notation by

$$
\begin{aligned}
& B_{m} * L=m\left(C_{m, s} * L\right) R^{m-1} \\
& A_{m} * L=-m\left(C_{m, c} * L\right) R^{m-1}
\end{aligned}
$$

The key step in this method is first to draw a circle, which is centered at and perpendicular to a prescribed reference trajectory. The OPERA-3D command CIRCLE is then employed to compute the radial component $B_{x} * t_{y}-$ $B_{y} * t_{x}$ or the azimuthal component $B_{x} * t_{x}+B_{y} * t_{y}$ of the magnetic field on the circle. The data are fit to the Fourier series for harmonic contents. A command input file is developed to move the circle in desired steps along the entire length of the reference trajectory and to accomplish the calculation automatically. Note that $t_{x}$ and $t_{y}$ are the tangential unit vectors to lines in the OPERA-3D. Though $B_{r}$ and $B_{t}$ are also used in the OPERA-3D postprocessor for the radial and azimuthal components of the magnetic field, they represent different physical quantities if the reference trajectory is curved.

Figure 9 shows the quadrupole term of the field on a cylindrical surface of radius $R=8 \mathrm{~cm}$ along the entire reference track. The reference track here consists of a straight segment for the upstream region from $z=-150$ to $z=0 \mathrm{~cm}$, an arc segment for the hard edge magnet from $z=0$ to $z=244.208 \mathrm{~cm}$, and a straight segment for the downstream region with a length of $200 \mathrm{~cm}$. The integrated skew quad term is $A_{2} * L=-0.0215 \mathrm{~T}$-m, resulting in an 


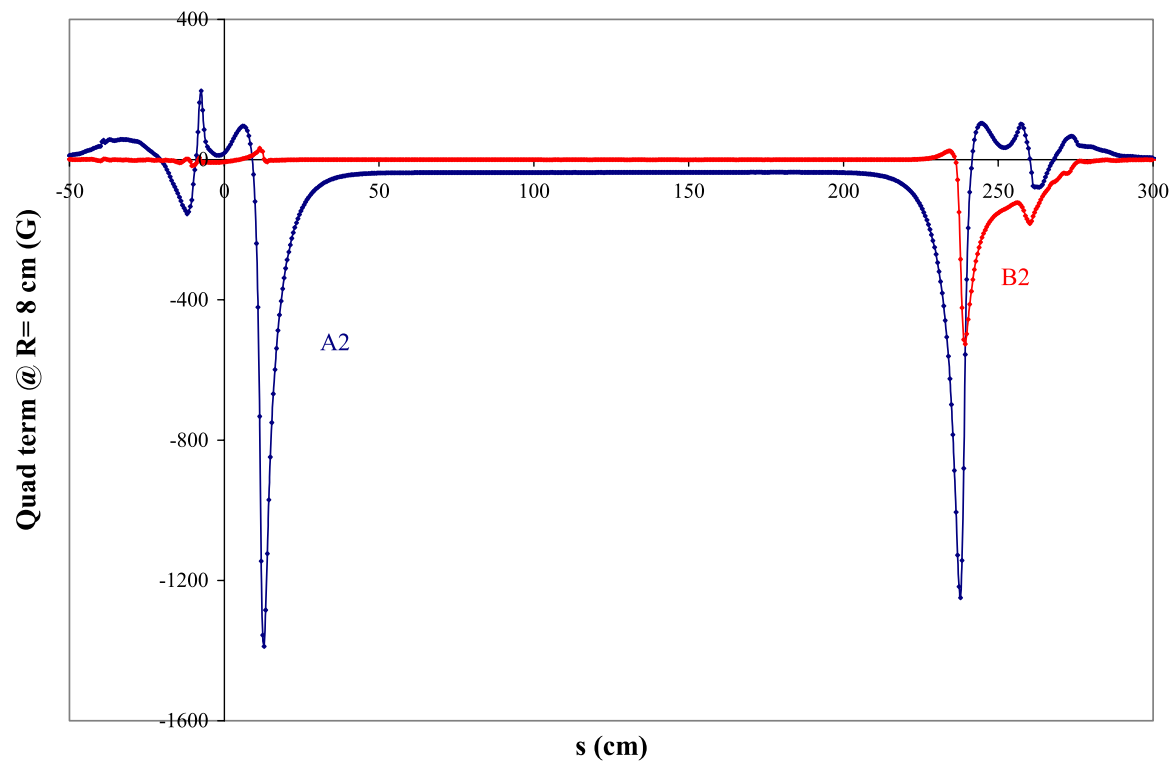

FIG. 9. (Color) Quad term of the field on a cylindrical surface surrounding a reference track.

integrated gradient of 0.269 T. In comparison with the result from the patch rotation method, the difference is less than $0.5 \%$. The integrated dipole term from this method is $B_{1} * L=-1.6926 \mathrm{~T}-\mathrm{m}$, yielding a bending angle of $17.14^{\circ}$ for a $1 \mathrm{GeV}$ proton. The integrated skew quad accounts for 127 units, which is fairly close to that from the patch rotation method. More harmonic data from this method are also listed in Table II.

At the magnet center, where the local coordinate system origin is $(9.064,0,123.428)$ and the Euler's angles are $(0$, $8.4,0$ ), the magnetic field on a circle perpendicular to the reference track contains a dipole term $C_{1}=6687.92 \mathrm{G}$ and a skew quad term $A_{2}=-36.90 \mathrm{G}$, which accounts for 55.2 units. The skew quad term everywhere in the central region of the magnet is very close to this value. This is too high for a transport line magnet with only twodimensional $(x$ and $y)$ field distributions.

\section{Dipole field on grids}

This method calculates the bending field along different, parallel trajectories and fits the results to a harmonic notation. The method mimics a flip coil measurement in experiments. The trajectories form a grid on a transverse cross section of the magnet aperture, as shown in Fig. 10. There are a total of 21 positions in the grid for the integrated $B_{y}$ calculation. The trajectory for each grid position consists of three parts: two straight lines before and after the hard edge magnet and one arc inside the hard edge magnet. All the trajectories are in parallel with the reference trajectory.

In cylindrical coordinates, a two-dimensional bending field $B_{y}$ can be expressed as [7]

$$
B_{y}(r, \theta)=\sum_{m=1}^{\infty} C_{m}\left(\frac{r}{R}\right)^{m-1} \cos \left[(m-1) \theta-m \alpha_{m}\right] .
$$

A five term presentation of the field is

$$
\begin{aligned}
B_{y}(r, \theta)-C_{1} \cdot \cos \left(\alpha_{1}\right)= & C_{2}\left(\frac{r}{R}\right) \cos \left(\theta-2 \alpha_{2}\right)+C_{3}\left(\frac{r}{R}\right)^{2} \cos \left(2 \theta-3 \alpha_{3}\right)+C_{4}\left(\frac{r}{R}\right)^{3} \cos \left(3 \theta-4 \alpha_{4}\right) \\
& +C_{5}\left(\frac{r}{R}\right)^{4} \cos \left(4 \theta-5 \alpha_{5}\right)+\cdots
\end{aligned}
$$

These equations also apply to the integrated bending field, in which the field amplitude terms have the dimension of T-m rather than T. It can be seen that the term $C_{1} *$ $L \cdot \cos \left(\alpha_{1}\right)$ can be obtained by measuring the bending field on the reference trajectory $(r=0)$. Figure 11 plots the lefthand side values of Eq. (12), $\Delta B_{y} * L=B_{y}(r, \theta) * L-$ $C_{1} * L \cdot \cos \left(\alpha_{1}\right)$, at different grid points based on the simulation calculation. The figure also shows the BNL test data as discussed in the next subsection. A best fitting of the simulation data by the least square method in MATHEMATICA [10] leads to the integrated field amplitude $C_{m} * L$ and phase angle $\alpha_{m}$. The integrated normal and skew terms can then be found from Eq. (5). This method yields an integrated skew quad term $A_{2} * L=$ $-0.0221 \mathrm{~T}-\mathrm{m}$ from the simulation data. This amplitude is larger by about $3 \%$ than that obtained with the patch 


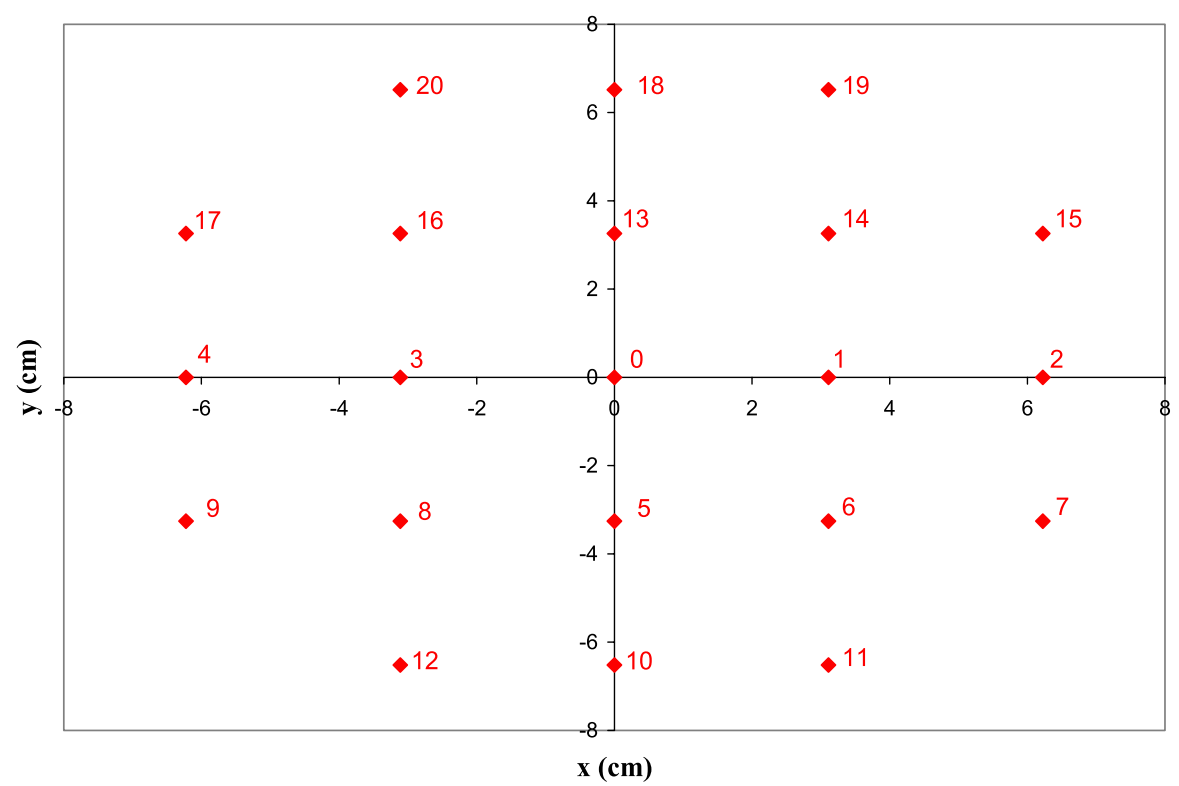

FIG. 10. (Color) Grid points for the dipole field method.

rotation method and the surface field analysis. The other harmonics from the fitting are also listed in Table II.

It should be noted that this method is not as accurate as the other two above. The main problem is that the fitting in MATHEMATICA is based on the least square method rather than the orthogonality condition. The fit results do depend on the number of terms used in Eq. (11), especially for higher harmonics. Our practice shows that the quadrupole term remains roughly the same when two to six terms are used for the fitting. Though it is not as accurate and reliable as the patch rotation method and the surface field analysis, the method of the dipole field on grids is developed here mainly for processing the BNL test data as described below.

\section{Comparison with measurements}

The ELS extraction line was measured at BNL with a flip coil on the same grids pattern as in Fig. 10. The BNL test data are plotted in Fig. 11. The same fitting method yields an integrated skew quadrupole term $A_{2} * L=$ $-0.0166 \mathrm{~T}-\mathrm{m}$. The other harmonics from fitting the measurement data are listed in Table II.

The integrated skew quad term in the BNL measurement data is only $75 \%$ of the value from the 3D simulation. This

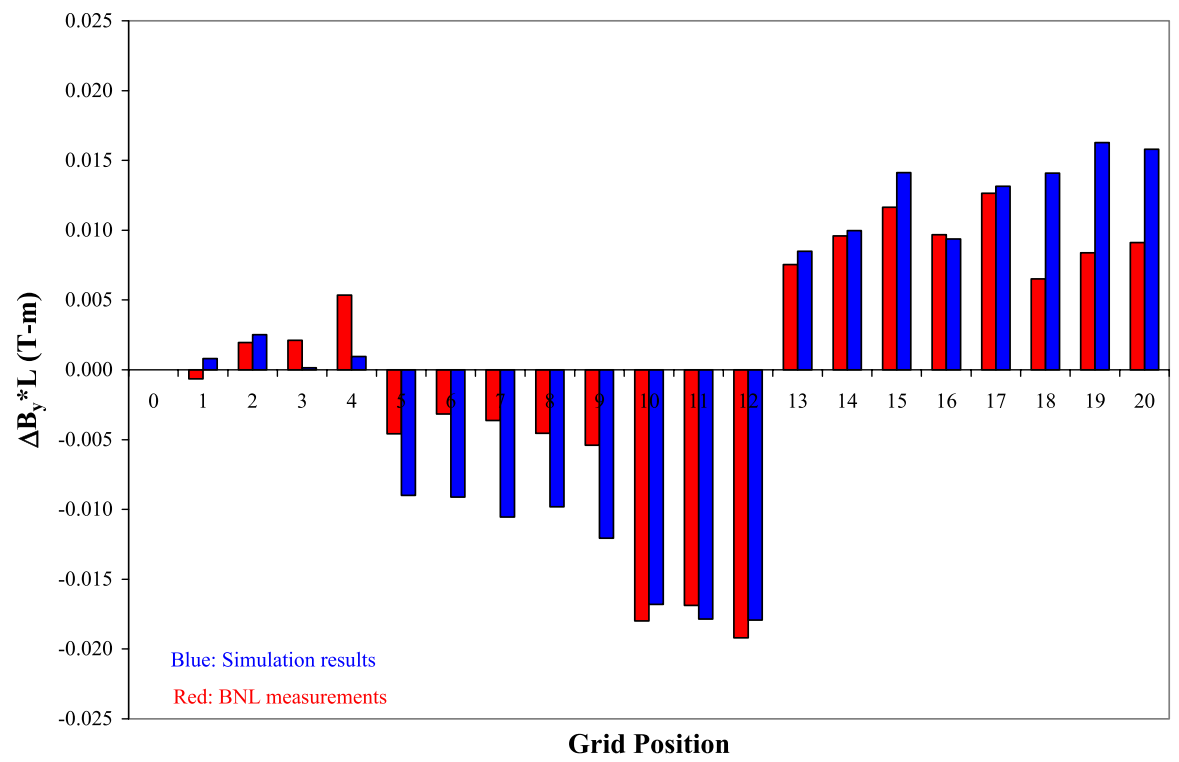

FIG. 11. (Color) Simulation and test data from the integrated dipole field on grids. 
large discrepancy between the 3D simulation and the BNL measurement is likely explained by measurement errors. In measurements with a flip coil following a curved reference trajectory, it is very difficult to achieve a relative accuracy of $0.25 \%$ [11], which corresponds to an error of more than one vertical division in Fig. 11. Although this discrepancy exists, the measurement data indeed show an integrated skew quad term of about 100 units, which is still 1 order of magnitude larger than that usually required for a transfer line magnet.

\section{ORIGIN OF THE LARGE SKEW QUAD TERM}

The integrated skew quad term in the ELS has two constituents as shown in Fig. 9. The first one comes from the central region $(s=50$ to $200 \mathrm{~cm}$ ) of the magnet, which accounts for $25.8 \%$ of the total. This is due to the up-down asymmetry of the pole tip and coil configuration and unoptimized compensation by the longitudinal shims. The second one results from the end effect of the magnet, which uses a longer septum plate to shield the circulating beam from the fringe field of the dipole below. This structure naturally contributes to the skew quad term, which accounts for $38.8 \%$ of the total at the magnet entrance and $35.4 \%$ of the total at the magnet exit. Thus, we investigate the two constituents separately, as described below.

\section{A. Effect of longitudinal shims on skew quad term}

We use OPERA-2D to study the effect of longitudinal shims on the skew quad term in the central region of the magnet. A half cross section of Fig. 3(b) is built in a 2D model, where the circulating beam aperture is left out. Figure 12 shows a zoom-in view of the ELS pole region in the 2D model. The magnetic flux lines indicate the field uniformity. Because of the up-down asymmetry of the pole tip and the coil configuration, the magnetic flux lines originated from the pole tip tend to spread horizontally when they go up to the septum plate. They are denser just above the pole tip than in the upper region of the gap. This creates a field gradient in the vertical direction, which is a skew quad term. It is common practice to employ the shims for compensation of this field nonuniformity in the vertical direction in order to minimize the skew quad term. Unfortunately, the original design of the shim, which has a trapezoidal cross section with a bottom width of $4.496 \mathrm{~cm}$, a top length of $3.683 \mathrm{~cm}$, and a height of $1.125 \mathrm{~cm}$, does not optimize the compensation and leaves a large skew quad term in the central region of the magnet.

In Fig. 13 we plot the magnetic field component $B_{y}$ of the original design along a vertical line from $y=-8$ to $y=8 \mathrm{~cm}$ at $x=0$ (blue curve). It is clear that there is a gradient in the $y$ direction and the maximum difference in $B_{y}$ is about $50.8 \mathrm{G}$. On a semicircle of radius $R=8 \mathrm{~cm}$ and centered at $x=y=0$, as shown by the dots in Fig. 12, we calculate the radial component $B_{r}=B_{x} * n_{v x}+B_{y} * n_{v y}$ or the azimuthal component $B_{\theta}=B_{x} * t_{v x}+B_{y} * t_{v y}$ of the magnetic field. The data are decomposed into the Fourier harmonics, which are listed in Table III. The total dipole field is $-6692.30 \mathrm{G}$, while the skew quad term accounts for $-37.79 \mathrm{G}$, corresponding to 56.5 units. This

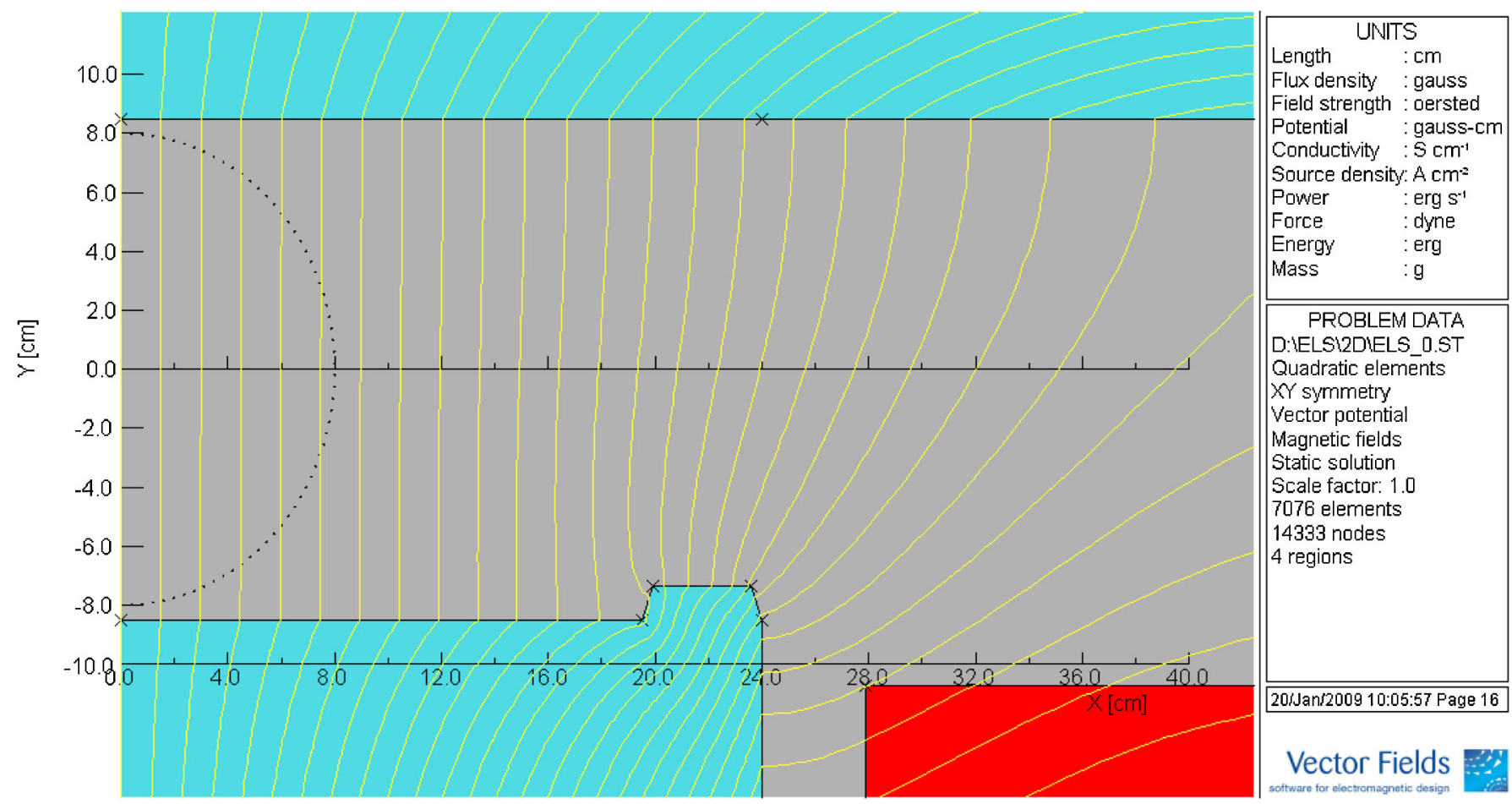

FIG. 12. (Color) The central area of a 2D ELS model. 


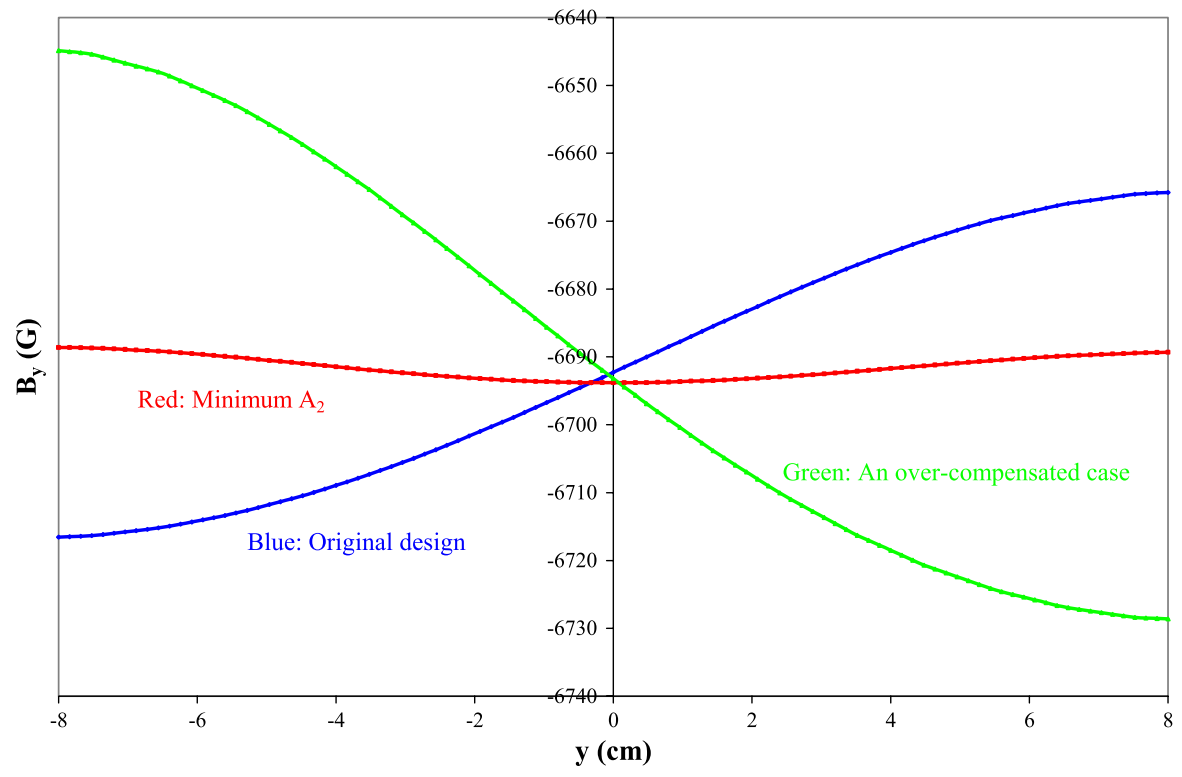

FIG. 13. (Color) $B_{y}$ vs $y$ at $x=0$ for $y=-8$ to $+8 \mathrm{~cm}$ in three different shim geometries.

is fairly close to the result of 55.2 units for the central region of the magnet as obtained in the 3D model as shown in Fig. 9. Note that $n_{v x}$ and $n_{v y}$ are the normal unit vector to a line, and $t_{v x}$ and $t_{v y}$ are the tangential unit vector to a line in OPERA-2D.

The skew quad term in the ELS central region is very sensitive to the shim geometry. With the 2D code we have generated various skew quad terms for different shim cross sections. Note that the specific shim cross section for a desired skew quad term is not unique. One may use the traditional method of trial and error to find a solution, or may employ the OPERA-2D OPTIMIZER to speed up calculations. In Fig. 14 we plot three shim cross sections, including the original design. The field uniformity $B_{y}$ vs $y$ for the other two shim geometries is also shown in Fig. 13, and the corresponding harmonic contents are listed in Table III.

The red shim geometry has a trapezoidal cross section with its bottom width of $7.431 \mathrm{~cm}$, height of $1.143 \mathrm{~cm}$, and $18^{\circ}$ on its two edges. The magnetic field $B_{y}$ along a line from $y=-8$ to $y=8 \mathrm{~cm}$ at $x=0$ shown in Fig. 13 is quite flat and is almost an even function with respect to $y=$ 0 , indicating that no skew quad term exists. Indeed, a
Fourier analysis of the data on the semicircle yields a skew quad term of $-0.002 \mathrm{G}$, a negligible amount.

The green shim geometry also has a trapezoidal cross section with its bottom width of $5.842 \mathrm{~cm}$, height of $2.54 \mathrm{~cm}$, and still $18^{\circ}$ on the two side edges. It would produce a field distribution along the $y$ axis, as plotted in Fig. 13. The field at the bottom of the pole tip $(y=-8 \mathrm{~cm})$ is lower than that on the top $(y=8 \mathrm{~cm})$, while it is the opposite in the original design. The maximum difference in $B_{y}$ is $83.7 \mathrm{G}$. The same Fourier decomposition of the field data on the semicircle yields a skew quad term of $+61.97 \mathrm{G}$. Note that the sign changes from the original case. This is the consequence of overcompensation by the shim. The other higher harmonics are listed in Table III. The usefulness of this shim geometry will be clear in Sec. VI.

\section{B. Effect of magnet ends on skew quad term}

In the ELS design, the septum plate is extended out of the pole-tip end by about $20 \mathrm{~cm}$ for shielding the circulating beam from the fringe field of the dipole below. This structure naturally contributes to a skew quad term since there is an up-down field gradient in the fringe region. The

TABLE III. Harmonic amplitudes (in G) in three different shim cross sections.

\begin{tabular}{rrrrrrr}
\hline \hline & \multicolumn{2}{c}{ Original design } & \multicolumn{2}{c}{ Minimum $A 2$} & \multicolumn{2}{c}{ Overcompensated } \\
$m$ & \multicolumn{1}{c}{$B_{m}$} & \multicolumn{1}{c}{$A_{m}$} & \multicolumn{1}{c}{$B_{m}$} & \multicolumn{1}{c}{$A_{m}$} & $B_{m}$ & \multicolumn{1}{c}{$A_{m}$} \\
\hline 1 & -6692.30 & 0.00 & -6693.82 & 0.00 & -6693.32 & 0.00 \\
2 & 0.00 & -37.79 & 0.00 & $-2.02 \times 10^{-3}$ & 0.00 & +61.97 \\
3 & -2.51 & 0.00 & -10.77 & 0.00 & -14.44 & 0.00 \\
4 & 0.00 & -13.94 & 0.00 & -1.51 & 0.00 & 21.36 \\
5 & -1.86 & 0.00 & -7.69 & 0.00 & -10.44 & 0.00 \\
\hline \hline
\end{tabular}




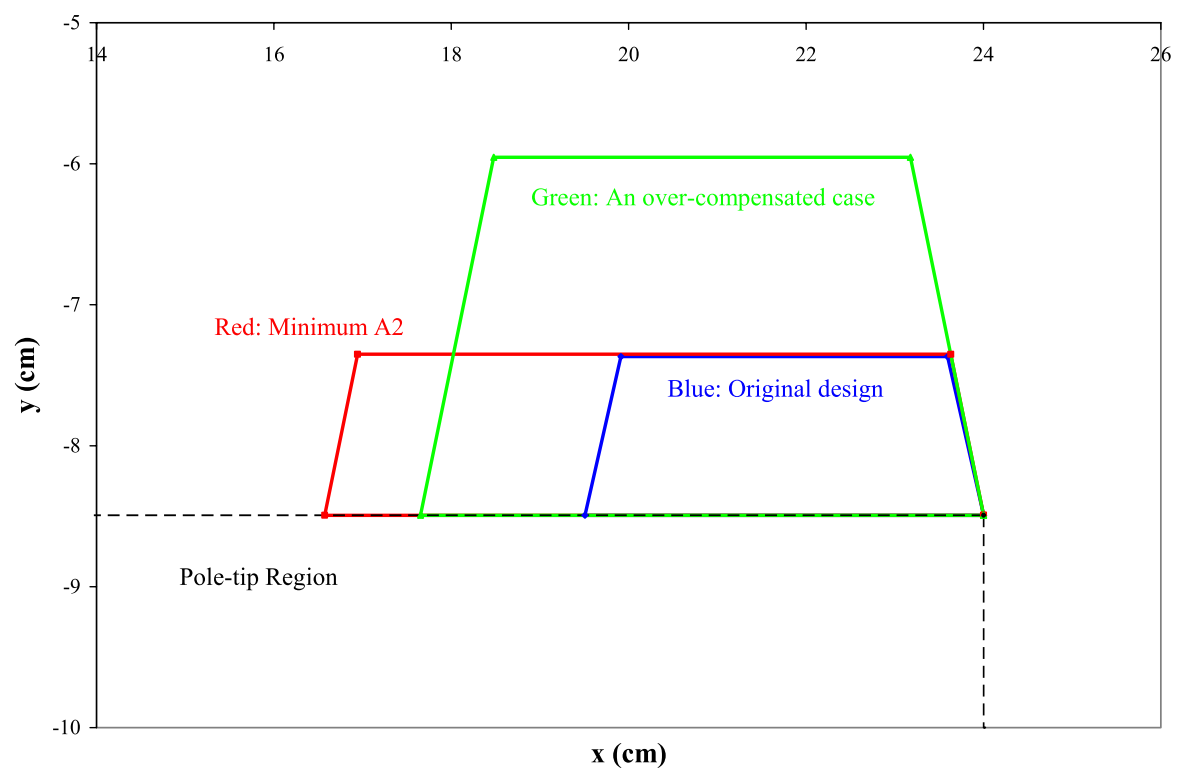

FIG. 14. (Color) Cross sections of three longitudinal shims.

magnetic flux lines coming out of the pole-tip end spread out rapidly when they go up towards the septum plate. This is demonstrated in Fig. 15, which shows the magnetic field component $B_{y}$ versus $y$ at $x=0$ and $z=12.116 \mathrm{~cm}$. The vertical line picked here for the plot is just at the septum dipole entrance, corresponding to the $s$ position for the first big peak in Fig. 9. The field component $B_{y}$ has an amplitude close to $1.1 \mathrm{~T}$ at $y=-8 \mathrm{~cm}$. The field amplitude decreases going up in the $y$ direction, and it drops to about $0.54 \mathrm{~T}$ at $y=8 \mathrm{~cm}$. The field gradient is most profound in the lower half of the gap with a $B_{y}$ difference of about $0.5 \mathrm{~T}$ from $y=0$ to $y=-8 \mathrm{~cm}$, while the field is relatively flat in the upper half of the gap with a $B_{y}$ difference less than $0.05 \mathrm{~T}$ from $y=8$ to $y=0 \mathrm{~cm}$. The $B_{y}$ versus $y$ plot at the same $z=12.116 \mathrm{~cm}$ and other $x$ values from -8 to $8 \mathrm{~cm}$ is very similar to Fig. 15.

A chamfer angle at the pole-tip end would alleviate the problem, but it was overlooked in the original design. For the existing ELS, we have noticed that there is a gap of more than $10 \mathrm{~cm}$ between the pole-tip end and the coil.

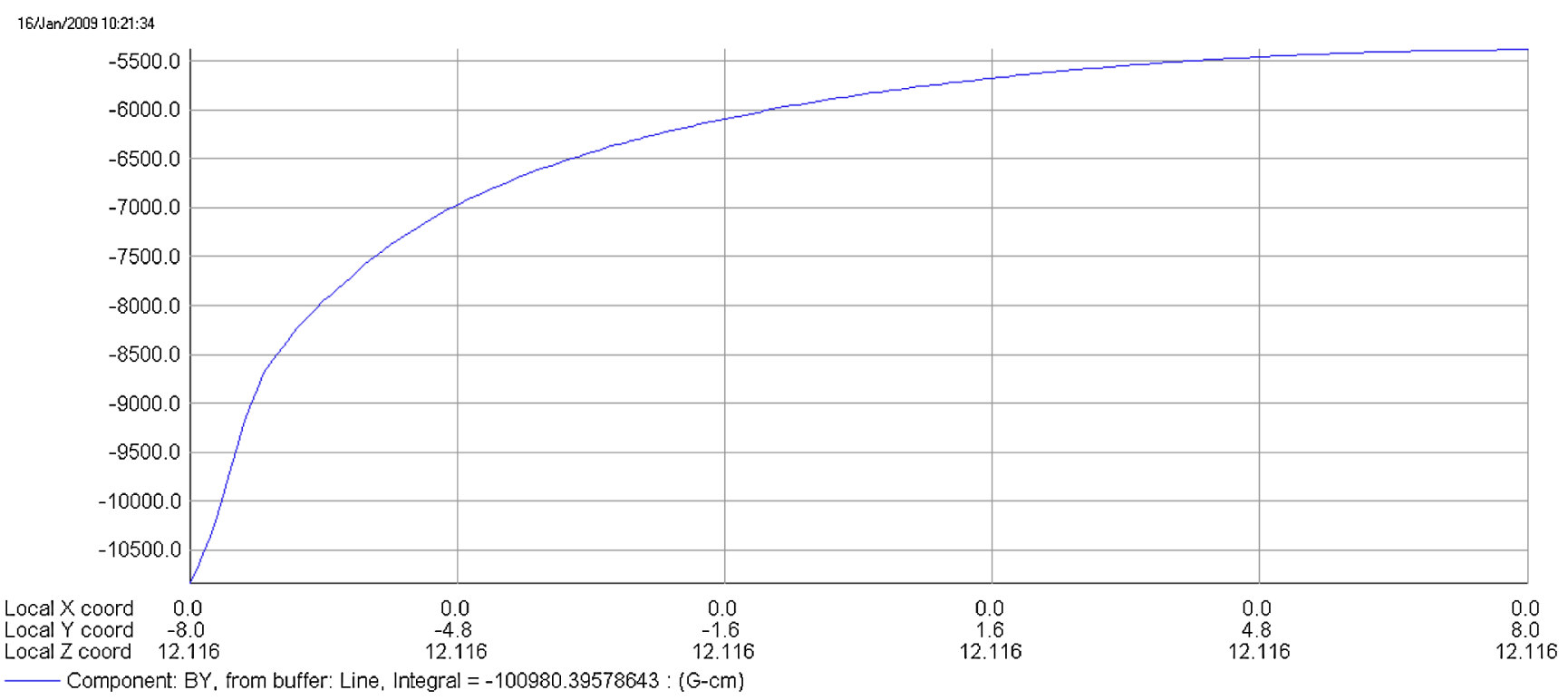

FIG. 15. (Color) $B_{y}$ vs $y$ at $x=0$ and $z=12.116 \mathrm{~cm}$. 


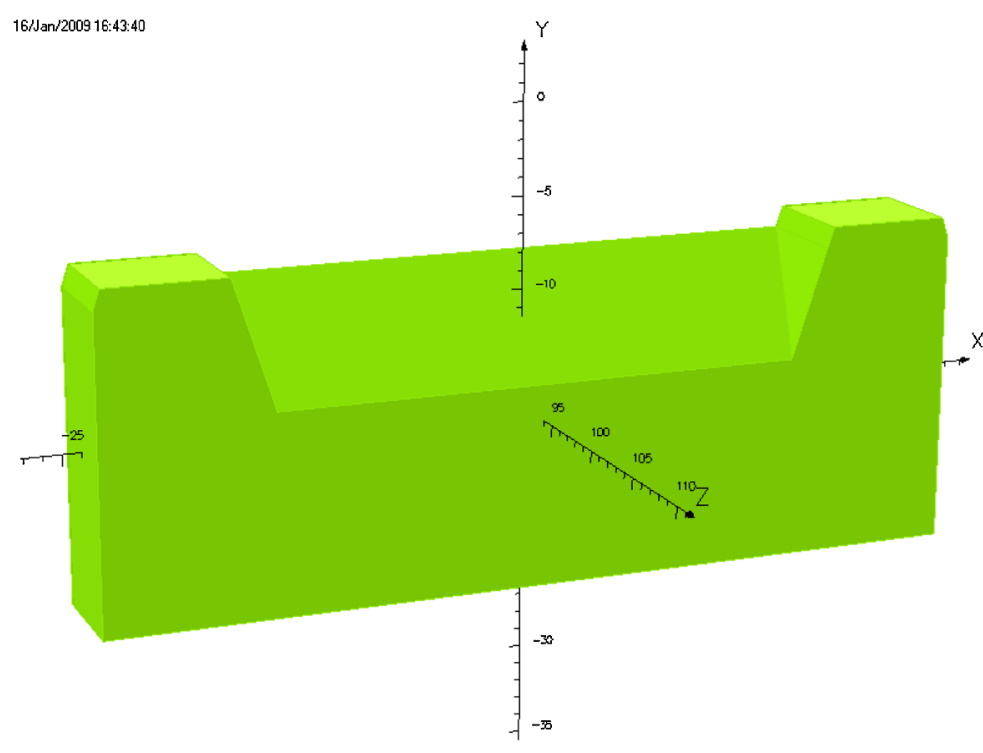

Vector Fields

FIG. 16. (Color) A $z$ block being attached to the pole-tip end for changing the integrated skew quad term.

This space can be utilized for the insertion of $z$ blocks with appropriate chamfer angles. In addition, the longitudinal shims in the magnet central region should be extended to the end of the $z$ block with specially trimmed edges. Such a $z$ block is illustrated in Fig. 16. This method could reduce the vertical field gradient in the dipole entrance and exit, as well as the integrated skew quad term in the whole fringe region.

In order to demonstrate the usefulness of the $z$ blocks with appropriate chamfer angles, we have built simplified ELS models, which are straight, shorter, and have no circulating beam aperture with associated end structures.
This greatly speeds up the iteration process for obtaining desired $z$ block geometries in simulation. Figure 17 shows the skew quad term in the magnetic field on a cylindrical surface of radius $R=8 \mathrm{~cm}$ in the magnet entrance fringe region for three different pole-tip and $z$-block configurations. In the figure, the pole-tip end is at about $z=12 \mathrm{~cm}$ and the septum plate end is at about $z=-8 \mathrm{~cm}$. The blue curve is for the original design where there is no $z$ block attached. Note that this curve is not exactly the same as the one in Fig. 9 because it comes from the simplified straight model rather than the real model. An integration of $A_{2}$ from $z=-100$ to $60 \mathrm{~cm}$ yields $-7300 \mathrm{G}-\mathrm{cm}$. The contribution

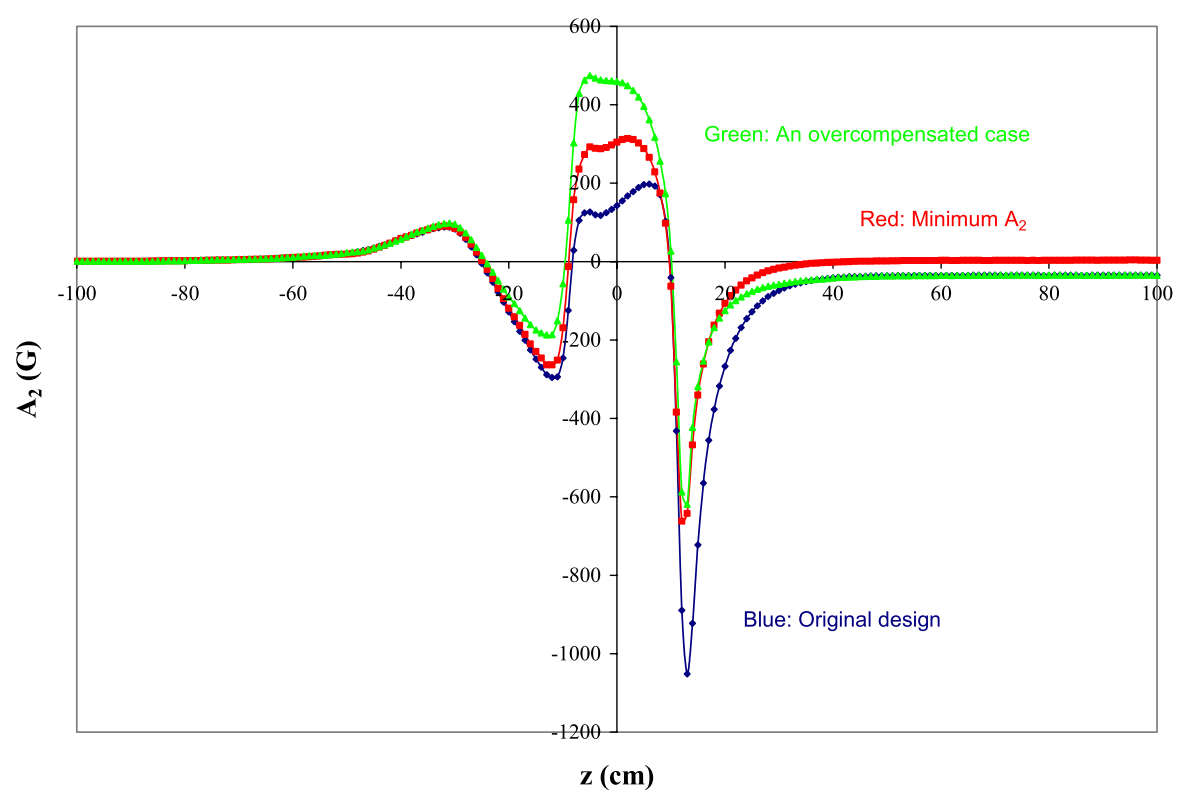

FIG. 17. (Color) Skew quad term along a cylindrical surface of radius $R=8 \mathrm{~cm}$ for three cases. 
from the longitudinal shims in the region from $z=12$ to $z=60 \mathrm{~cm}$ is about $-1700 \mathrm{G}-\mathrm{cm}$. Thus, the integrated skew quad term due to the fringe field is about $-5600 \mathrm{G}-\mathrm{cm}$.

The red curve shows the results from a modified simple model, where the longitudinal shims take the cross section as the red one in Fig. 14 and a $z$ block is attached to the pole-tip end. The integrated skew quad term from $z=$ -100 to $60 \mathrm{~cm}$ is now $-5.5 \mathrm{G}-\mathrm{cm}$. Since the new shims do not contribute to the skew quad term inside the magnet, the $-5.5 \mathrm{G}-\mathrm{cm}$ results from the cancellation of the skew quad terms in the fringe region. This is a significant reduction for one end of the magnet. By carefully trimming the $z$ block, it is possible in principle to eliminate completely the integrated skew quad term.

The green curve shows the results from another simple model where the longitudinal shims remain as in the original design and the $z$ block takes a different geometry. The integrated skew quad term in the same region is $+2737 \mathrm{G}$. This is an overcompensated case by the $z$ block that changes the sign of the integrated skew quad. It turns out that this positive amount of the skew quad in the fringe region would cancel the negative amount of the skew quad term in the central region due to the original shims, resulting in an almost vanishing integrated skew quad term over the entire length of the simplified septum model.

\section{MINIMIZATION OF INTEGRATED SKEW QUAD TERM}

The analysis results described in the previous section suggest that there are three possible options to modify the existing ELS in situ in order to minimize its integrated skew quad term. The first option would be to replace the existing longitudinal shims by the green one in Fig. 14, which would produce a positive skew quad term in the central region to compensate the negative ones from the two ends. The second option would be to insert the $z$ blocks between the pole-tip end and the coil to produce a positive integrated skew term, which would cancel the contribution from the magnet central region. These $z$ blocks should be similar to the one that yields the green curve in Fig. 17. The third option would be to employ the red shim geometry in Fig. 14, which would make the skew quad term uniformly vanishing in the magnet central region. In addition, the $z$ blocks would be added to the pole-tip ends to produce a vanishing integrated skew quad term in the fringe region, similar to the red curve in Fig. 17.

In principle, all three options should lead to a very small integrated skew quad term in a modified ELS. Nevertheless, the second and third options with the insertion of $z$ blocks are more involved in practice. The simplified ELS models with the $z$ blocks contain a significantly higher integrated sextupole. The insertion of the $z$ blocks, which is equivalent to an extension of the pole-tip length, would increase the fringe field contribution to the circulating beam line and so more shielding is required. Furthermore, it is very time consuming to optimize the $z$-block geometry in realistic 3D ELS models, where the $z$ blocks at the magnet entrance and exit should be slightly different. We have so far produced a number of modified, realistic ELS models with the $z$ blocks, and their overall performance is not as good as that from the first option. Therefore, our proposed modification is the first option and it is described in detail below.

A full 3D modified ELS model of the first option, which requires only a change of the longitudinal shims, has been constructed. The skew quadrupole term of the magnetic field on a cylindrical surface of radius $8 \mathrm{~cm}$ is shown in

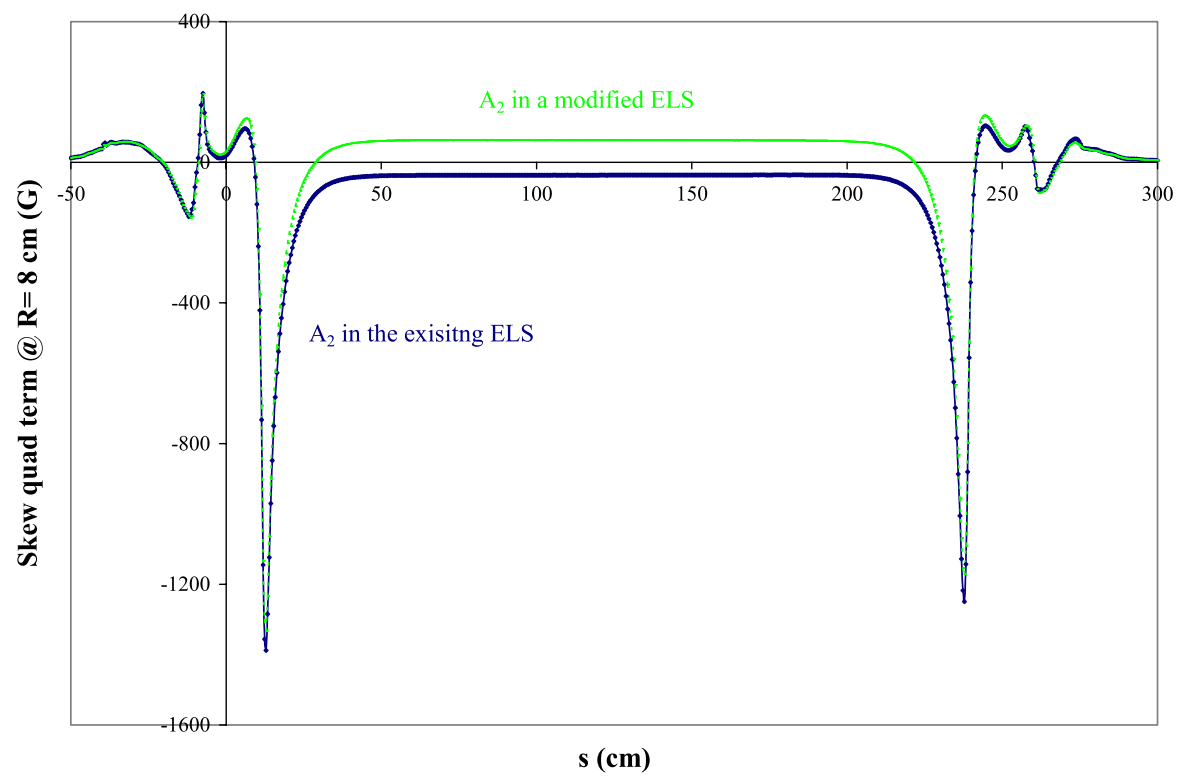

FIG. 18. (Color) Skew quad term of the field on a cylindrical surface surrounding a reference track. 
Fig. 18. For comparison purposes, we also plot the result for the existing ELS from Fig. 9. The integrated skew quad term for the modified ELS is now $0.00054 \mathrm{~T}-\mathrm{m}$, or 3.2 units. The corresponding integrated gradient is $0.0067 \mathrm{~T}$. This is about 40 times smaller than that in the existing magnet. It is expected that the integrated skew quad term would be even smaller when an adjacent quadrupole downstream of the ELS is taken into account, as discussed later.

The integrated higher harmonics for the existing ELS are listed in Table II. They are 16.1, 29.3, and 0.4 units for the sextupole, octupole, and decapole, respectively. For the modified ELS from the first option, the integrals of the sextupole term and octupole term are 1.4 and 19.6 units, respectively. Both of them are smaller than those in the original design due to the cancellation between the central region and the fringe region. The integrated decapole in the modified one is 10.3 units, larger than before. We do not expect any problem for the extracted beam from this slightly higher decapole term.

\section{EXTRACTED BEAM PROFILE THROUGH ELS}

A skew quadrupole has its focusing and defocusing planes rotated by $45^{\circ}$ with respect to the focusing and defocusing planes of a normal quadrupole. Thus, the skew quadrupole distorts the beam profile in a lattice conventionally consisting of all the normal quadrupoles. In order to verify the effect of the skew quad term on the beam profile, we study the extracted beam trajectories and profiles through the ELS before and after the modification of the magnet. We launch a laminar beam of an elliptical cross section without space charge upstream of the magnet. The initial particles move in the direction of the $z$ axis. We select a reference particle on the axis and 36 more on the surface of the ellipse. Since the integrated ELS bending field in the model is slightly higher than the nominal design value, the proton energy is set to $1.0298 \mathrm{GeV}$ in order for the reference particle to follow the nominal design trajectory. OPERATRACK command is employed to calculate the particle trajectories through the ELS extraction channel as shown in Fig. 19. At the end of the trajectories, we create a Cartesian patch, which is perpendicular to the reference trajectory. The intersection of all the particle trajectories on the patch is recorded. This yields the extracted beam profile downstream of the ELS.

Figure 20 shows the extracted beam profiles from the study. The dotted blue curve is the beam profile at $z=$ $-150 \mathrm{~cm}$ from the ELS entrance. It is a perfect, right ellipse, which should be the case in the nominal design and operation. The red curve indicates the extracted beam profile from the existing septum at its output, which is about $194.2 \mathrm{~cm}$ from the ELS exit. It is clear that the extracted beam profile is distorted and looks to be tilted. The test particle upstream at $A(4,0)$ on the elliptical surface is moved to $A^{\prime}$ downstream of the existing ELS. The angle between $O A$ and $O A^{\prime}$ is about $9.7^{\circ}$. The beam profile tilt appears to be even larger than this angle. This extracted beam profile resembles that observed in the early experiment, as shown in Fig. 2, though there are differences due to different beam parameters, location of evaluation, etc.

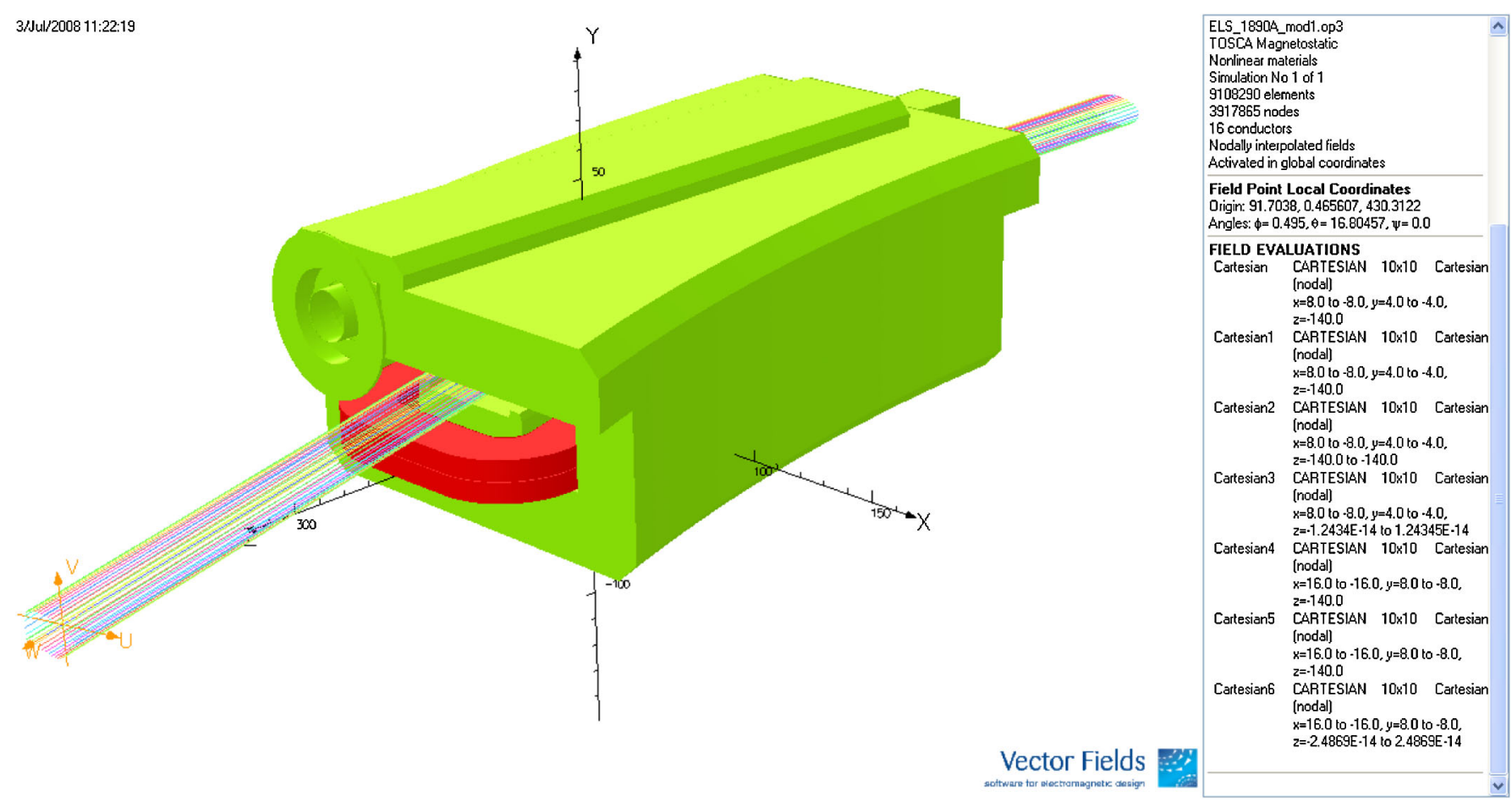

FIG. 19. (Color) Trajectories of a laminar beam without space charge through the ELS. 


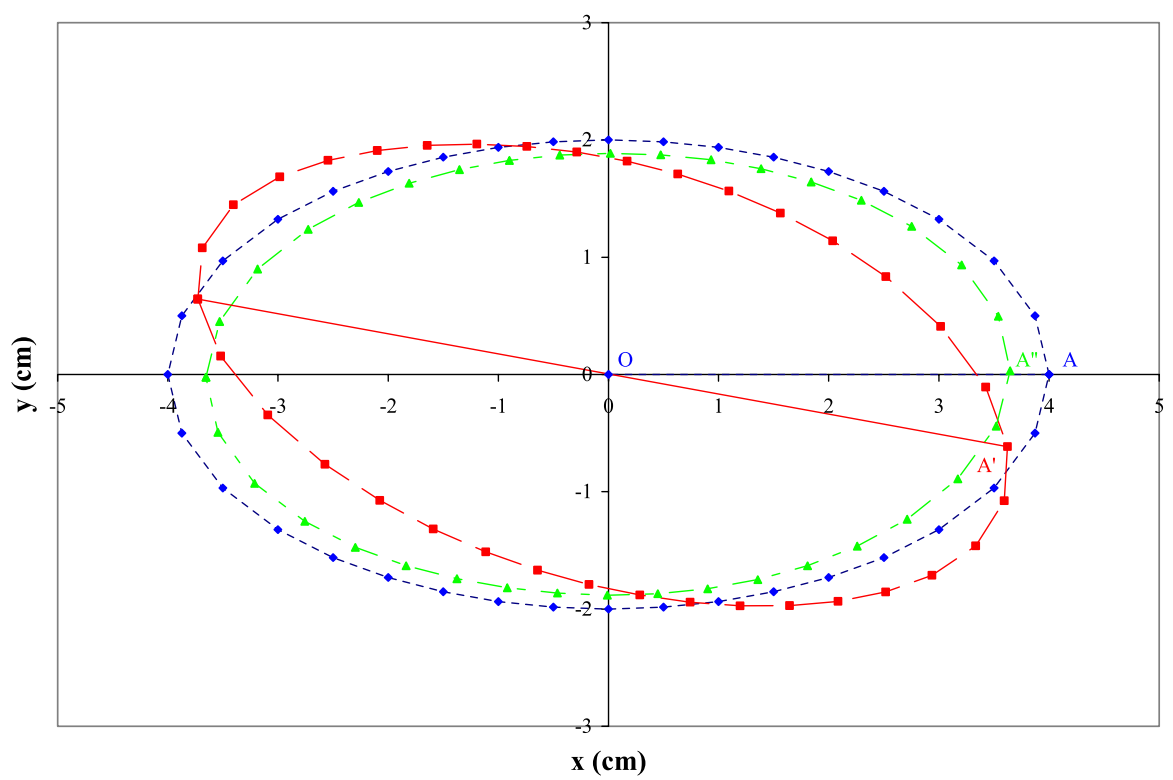

FIG. 20. (Color) Extracted beam profiles through the existing and modified ELS.

The green curve in Fig. 20 shows the extracted beam profile from the modified septum at the same output location. The test particle at $A$ is moved to the point $A^{\prime \prime}$. Very little beam profile tilt can be seen. Indeed, the extracted beam profile cross section is a fairly good ellipse. The profile tilt is corrected with the ELS modification.

\section{ELS INTERFERENCE WITH QUAD}

The magnetic interference in magnet assemblies has been studied in 3D simulations for the SNS project. The results from the quad doublet assembly just after the ELS (Fig. 1) were reported in [12]. For the interference study of a two magnet assembly in simulations, there are usually at least two models required for comparison purpose. One is for an individual magnet and another is for the two magnet assembly. Special caution should be taken to avoid artificial errors due to mesh generation in the two models. The best way to achieve this goal is that the same two magnet assembly model is used to simulate any individual magnet provided that the other magnet is presented by air material and zero-coil current [13].

The interference between the ELS and an adjacent quadrupole $30 \mathrm{Q} 44$ is investigated in a simulation model, consisting of the two magnets. As shown in Fig. 1, the quadrupole 30Q44 center is about $300 \mathrm{~cm}$ downstream of the ELS entrance. The closest distance between the surface of the ELS exit cap and the 30Q44 yoke is merely $22.261 \mathrm{~cm}$. It is expected that the quad 30Q44 affects the ELS extracted beam and vice versa. The simulation model contains about 15 million elements, and the postprocessor file occupies 4.2 GB memory.

The effect of the ELS on the parameters of 30Q44 in the study was reported in [14]. Below we briefly describe the main results that show the effect of 30Q44 on the ELS extracted beam in terms of the skew quad.

The existence of 30Q44 makes the magnetic flux lines originated from the ELS dipole region spread further to the quad yoke material. This is equivalent to increase the vertical field gradient in the ELS exit region. The detailed analysis shows that the total integrated skew quad term in the existing ELS alone is $-0.0215 \mathrm{~T}-\mathrm{m}$, while it is $-0.0226 \mathrm{~T}-\mathrm{m}$ in the ELS and 30Q44 model. This is an increase of about 5\%. As mentioned in Sec. VI, for the modified ELS the longitudinal shims with the green cross section shown in Fig. 14 could reduce the total integrated skew quad term to $+0.00054 \mathrm{~T}-\mathrm{m}$, a slightly positive value. Since the existence of $30 \mathrm{Q} 44$ increases the vertical gradient of the field in the ELS exit region, the total integrated skew quad goes in the negative direction in the modified ELS plus 30Q44 assembly. The numerical data show a total integrated skew quad term of $A_{2} * L=$ $-0.00033 \mathrm{~T}-\mathrm{m}$, corresponding to an integrated gradient of $G * L=0.0042 \mathrm{~T}$. This is a reduction of more than a factor of 60 in comparison with the original design.

\section{SUMMARY}

The SNS ring extraction Lambertson septum magnet contains a strong skew quad term, which causes a beam profile distortion on the target. We have performed 3D computer simulations with OPERA-3D/TOSCA to study the magnetic field quality in the magnet. The skew quad term is computed with various methods for better accuracy and reliability. The origin of the large skew quad term is investigated for the central region and the fringe region of the magnet. We find that the skew quad term could be made uniformly vanishing in the central region by appro- 
priate cross sections of the longitudinal shims; the integrated skew quad in the fringe region could also be made negligibly small with the $z$ blocks attached to the magnet pole-tip end. As a simpler alternative, a modification of the existing ELS for reducing its integrated skew quad is proposed, in which new longitudinal shims are employed to compensate the integrated skew quad term in the fringe region. The modified ELS could reduce the integrated skew quad by a factor of 40 , or more than 60 if the interference from an adjacent quad $30 \mathrm{Q} 44$ is taken into account. The study of the extracted beam trajectories and profile in the existing and modified ELS demonstrates the benefits of such a correction.

\section{ACKNOWLEDGMENTS}

ORNL/SNS is managed by UT-Battelle, LLC, for the U.S. Department of Energy under Contract No. DE-AC0500OR22725. The author would like to thank N. Tsoupas, W.Z. Meng, and A. Jain of BNL for discussions on the subject. The help from C. Riley and J. Simkin of Vector Field, England, in many details of OPERA code and simulation techniques is highly appreciated. The author would like to acknowledge the support and assistance in this work by G. Murdoch, D. Lousteau, J. Galambos, M. Plum, and T. Roseberry. The author also benefited from the work on the subject by S. Cousineau, J. Holmes, and T. Pelaia.
[1] N. Tsoupas, Y. Y. Lee, J. Rank, and J. Tuozzolo, in Proceedings of the Particle Accelerator Conference, Chicago, IL, 2001 (IEEE, New York, 2001), p. 3245.

[2] J. Rank, K. Malm, G. Miglionico, D. Raparia, N. Tsoupas, J. Tuozzolo, and Y. Y. Lee, in Proceedings of the 20th Particle Accelerator Conference, Portland, OR, 2003 (IEEE, New York, 2003), p. 2150.

[3] D. Raparia, in Proceedings of the 21st Particle Accelerator Conference, Knoxville, 2005 (IEEE, Piscataway, NJ, 2005), p. 553.

[4] M.A. Plum, in Proceedings of the 2007 Particle Accelerator Conference, Albuquerque, New Mexico, 2007 (IEEE, Albuquerque, New Mexico, 2007), p. 2603.

[5] OPERA-3D/TOSCA, Vector Fields, England. See http:// www.vectorfields.com/.

[6] K. Halbach, LBL Engineering Note, 1972.

[7] A. K. Jain, in Proceedings of CERN Accelerator School on Measurement and Alignment of Accelerator and Detector Magnets, Anacapri, Italy, 1997.

[8] M. Venturini, Ph.D. dissertation, University of Maryland at College Park, 1998.

[9] J. G. Wang, Phys. Rev. ST Accel. Beams 9, 012401 (2006).

[10] Stephen Wolfram, The MATHEMATICA Book (Wolfram Media/Cambridge University Press, Cambridge, UK; New York, NY; Melbourne, Australia, 1999), 4th ed.

[11] A. K. Jain (private communication).

[12] J. G. Wang, Phys. Rev. ST Accel. Beams 9, 122401 (2006).

[13] W.Z. Meng (private communication).

[14] J.G. Wang, in Proceedings of the 2007 Particle Accelerator Conference, Albuquerque, New Mexico, 2007 (Ref. [4]), p. 626. 